\title{
Working
}

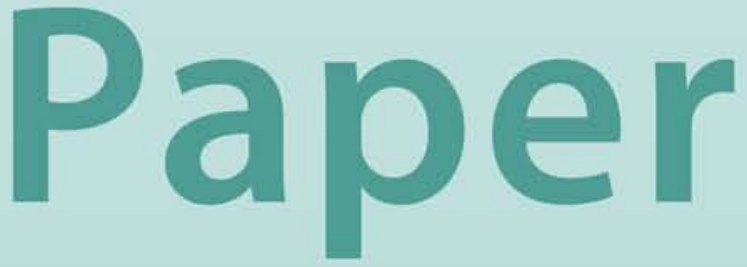




\section{Learning About Inflation Measures for Interest Rate Rules}

Marco Airaudo and Luis-Felipe Zanna 


\title{
IMF Working Paper
}

\author{
Research Department
}

\section{Learning About Inflation Measures for Interest Rate Rules}

Prepared by Marco Airaudo and Luis-Felipe Zanna*

\author{
Authorized for distribution by Andrew Berg
}

December 2010

\begin{abstract}

\section{This Working Paper should not be reported as representing the views of the IMF.}

The views expressed in this Working Paper are those of the author(s) and do not necessarily represent those of the IMF or IMF policy. Working Papers describe research in progress by the author(s) and are published to elicit comments and to further debate.
\end{abstract}

Empirical evidence suggests that goods are highly heterogeneous with respect to the degree of price rigidity. We develop a DSGE model featuring heterogeneous nominal rigidities across two sectors to study the equilibrium determinacy and stability under adaptive learning for interest rate rules that respond to inflation measures differing in their degree of price stickiness. We find that rules responding to headline inflation measures that assign a positive weight to the inflation of the sector with low price stickiness are more prone to generate macroeconomic instability than rules that respond exclusively to the inflation of the sector with high price stickiness. By this we mean that they are more prone to induce nonlearnable fundamental-driven equilibria, learnable self-fulfilling expectations equilibria, and equilibria where fluctuations are unbounded. We discuss how our results depend on the elasticity of substitution across goods, the degree of heterogeneity in price rigidity, as well as on the timing of the rule.

JEL Classification Numbers: C62, D83, E32, E52

Keywords: Learning; Expectational Stability; Interest Rate Rules; Multiple Equilibria; Determinacy; Multiple Sectors

Author’s E-Mail Address: ma639@drexel.edu, fzanna@imf.org

\footnotetext{
* The authors have benefited from comments and conversations with Mark Bils, David Bowman, Jim Bullard, Era Dabla-Norris, Jane Ihrig, Chris Erceg, Jordi Galí, Bruce McGough, Filippo Taddei and seminar participants at Collegio Carlo Alberto, the Federal Reserve Board, Paris X, the Catholic University of Milan, the Modigliani Workshop, the Society for Computational Economics, the "Learning in Macroeconomic Models" workshops at CFS, the ASSET 2007 Meeting and the 2010 Midwest Macroeconomics Meetings. James DiNicco provided useful research assistance. All errors remain ours. The views expressed in this paper are solely the responsibility of the authors and should not be interpreted as reflecting the view of the institutions to which they are affiliated.
} 
Table of Contents $\quad$ Page

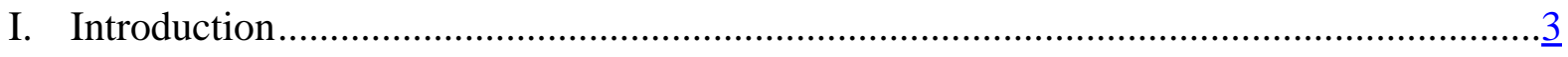

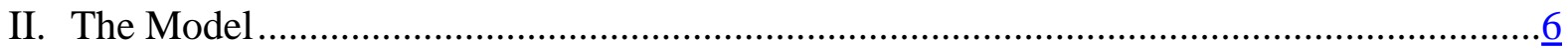

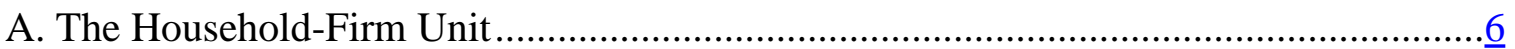

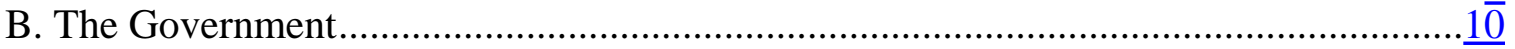

III. Equilibrium Determinacy and Stability under Learning................................................11

A. The Methodology ..........................................................................................

B. The Strong Dichotomy: A Flexible-Price and Sticky-Price Economy ........................13

C. The General Set-up: The Bils-Klenow Calibration ...................................................19

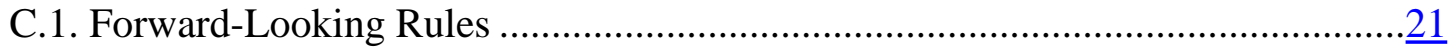

C.2. Other Timings for the Policy Rule ............................................................. $\frac{23}{26}$

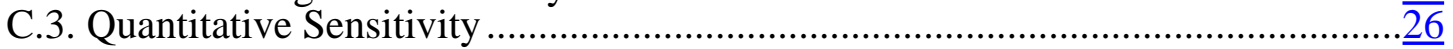

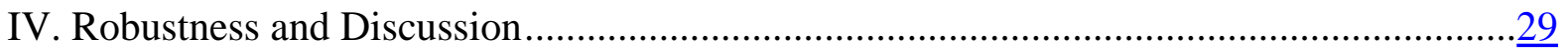

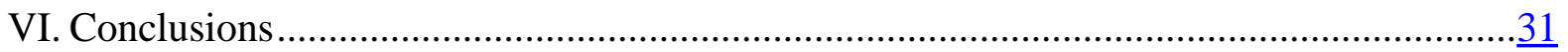

\section{Tables}

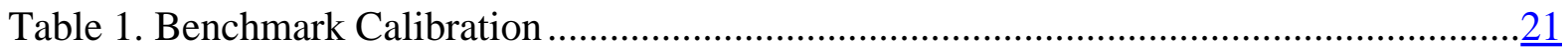

Table 2. Forward-Looking Rules: Determinacy and E-stability Bounds ..............................23

Table 3. Augmented Forward-Looking Rules: Determinacy and E-stability Bounds.............. $\underline{11}$

\section{Figures}

Figure 1. Forward-Looking Rules in the Dichotomous Economy.......................................17

Figure 2. Forward-Looking Rules in the General Set-up .............................................22

Figure 3. Contemporaneous Rules in the General Set-up..................................................24

Figure 4. Backward-Looking Rules in the General Set-up ..............................................25

Figure 5. Aggregate Price Stickiness and Heterogeneity Across Sectors............................. $\frac{27}{27}$

Figure 6. Varying the Share of Flexible-Price Goods.......................................................27

Figure 7. Forward-Looking Rules Responding to CPI Inflation .......................................28

Figure 8. Forward-Looking Rules that Respond to Output ..............................................

\section{Appendix}

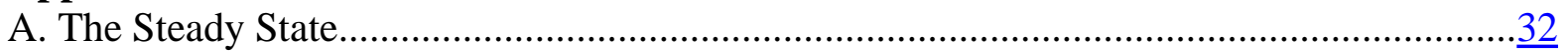

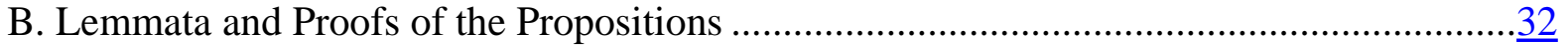

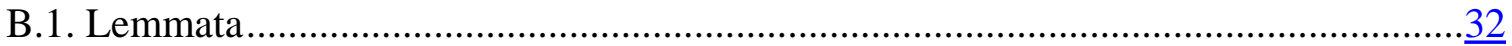

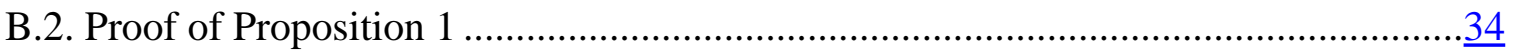

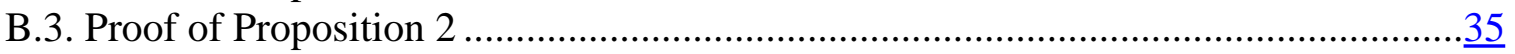

B.4. Proof of Proposition 3 ................................................................................

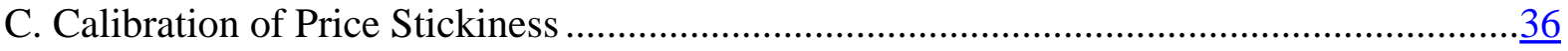

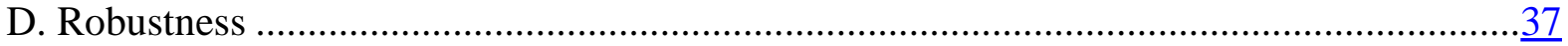

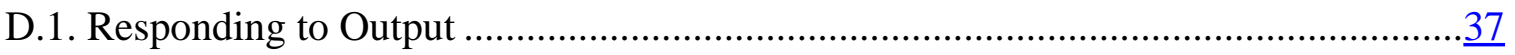

D.2. The Cash-In-Advance Timing ...................................................................

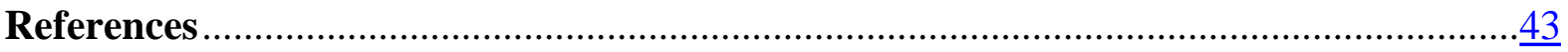




\section{Introduction}

The review by Klenow and Malin (2010) of the microeconomic literature of price setting concludes that goods are highly heterogeneous with respect to their degree of price stickiness. At one extreme are goods that change prices every quarter or even once a month, including food, energy, and airfares; and at the other extreme are services that change prices much less often than once a year. ${ }^{1}$ This microeconomic evidence seems to be confirmed by macroeconomic estimates. Bouakez et al. (2009), for example, estimate a multi-sector New-Keynesian model for the U.S. and cannot reject the hypothesis that some sectors - typically non-services - display flexible prices. In contrast, they also find that services is by far the most price-rigid sector in the U.S. economy.

This evidence may raise questions regarding the inflation measure that should be the target for monetary policy, including interest rate rules. In particular, central bankers may wonder whether monetary policy should respond to an inflation measure that puts some weight on inflation of flexibleprice goods or, instead, respond to a measure mainly based on the inflation of sticky-price goods. In fact, monetary policy discussions on the recent oil and food price shocks can be cast in these terms: should monetary policy respond to a headline inflation that includes the inflation of energy and food (flexible-price goods)? or should it respond to a core inflation measure that excludes the inflation of these goods? ${ }^{2}$

In this paper, we provide answers to these questions by selecting the inflation measure of an interest rate rule that is more prone to avert macroeconomic instability. By this we mean that the appropriate inflation measure of the rule should (i) shield the economy against fluctuations driven by self-fulfilling expectations $^{3}$ - Keynes' animal spirits - and (ii) ensure that even if agents are boundedly rational - in the sense of adaptive learning as proposed by Evans and Honkapohja (2001) - they can still learn the monetary policy targeted equilibrium so the economy will converge to it. ${ }^{4}$

Specifically, we develop a two-sector New-Keynesian DSGE model to study the determinacy and learning stability (E-stability) properties of Rational Expectations Equilibria (REE) for interest rate rules responding to inflation measures that differ in their degree of price stickiness. ${ }^{5}$ In the model, both sectors feature nominal price rigidities but one of them - which we call the flexible-price sector - faces low rigidities in comparison with the other sector - which we refer to as the sticky-price sector. If the aggregate inflation of the rule is expressed as a weighted average of the inflations of these two sectors,

\footnotetext{
${ }^{1}$ Bils and Klenow (2004) find, for instance, that about half of U.S. products display prices that last 4.3 months or less. This includes energy related goods and raw products, for which prices change about 54 percent of the months considered. Though more recent estimates of median price durations by Nakamura and Steinsson (2008) and Klenow and Kryvtsov (2008) are slightly higher, price stickiness heterogeneity appears to be undeniable.

${ }^{2}$ For a policy-maker discussion on the choice of price index see, for instance, Mishkin (2008) and Wynne (2008), among others.

${ }^{3}$ Woodford (2003) and references therein discuss how interest rate rules may induce endogenous fluctuations, in the presence of real indeterminacy (multiple equilibria).

${ }^{4}$ See Bullard and Mitra (2002) for a discussion on how interest rate rules may induce fundamental-driven equilibria that are not learnable.

${ }^{5}$ The concept of Expectational Stability (E-stability) for learning by Evans and Honkapoja (2001) is related to whether a given REE is aymptotically stable, when agents form forecasts using recursive least square learning algorithms and previous data from the economy.
} 
then a myriad of inflation measures can be considered by varying, for instance, the weight on the flexible-price inflation. By allowing the central bank pick this weight, we see the inflation measure as a policy variable, while focusing on rules that satisfy the Taylor principle; that is, active rules whereby the response coefficient to inflation is larger than one.

We find that active rules responding to an inflation measure which puts some weight on the flexibleprice inflation (a headline measure) are more prone to generate macroeconomic instability than rules that exclusively respond to sticky-price inflation (a core measure). As a result, policy makers should target the inflation measure of the truly sticky-price sectors.

Our conclusions follow from the analysis of several policy rule timings. We find that forward-looking rules that assign some positive weight to the expected flexible-price inflation are more prone to induce fundamental-driven equilibria that are not learnable and self-fulfilling expectations equilibria of the "sunspot" type that are learnable. Backward-looking rules that put some weight on the past flexibleprice inflation, on the other hand, can be also destabilizing, as they are more susceptible to generate explosive equilibria for which economic fluctuations are potentially unbounded. Only contemporaneous rules appear to guarantee a unique and learnable equilibrium, for any inflation measure; that is, even if the rule puts some weight on the current flexible-price inflation. But across these different timings, the safe choice to rule out potential sources of instability is to respond exclusively to the sticky-price inflation.

The intratemporal elasticity of substitution of goods in agents' preferences - in particular whether the goods are Edgeworth substitutes, neutral or complements - plays a key role in our results. Such elasticity determines the degree of comovement of the two sectoral inflation rates and, hence, affects the equilibrium response of the real interest rate to an adverse inflationary shock under different inflation measures. For instance, we show that equilibrium determinacy and learnability conditions are generally more stringent under the assumption of complementarity; and we identify a threshold for the weight on expected flexible-price inflation beyond which any active rule responding to the headline inflation always induces the non-learnability of the fundamental REE.

Our results hold under two different cases regarding the heterogeneity of price setting. First, we consider a strongly dichotomous economy with a fully flexible-price sector - which faces no price rigidities - and a sticky-price sector. This case allows us to derive analytical results and a straightforward intuition. In addition, this economy is analogous, on one hand, to the framework of Aoki (2001) and subsequent work in closed economies on optimal policy; ${ }^{6}$ while, on the other hand, it resembles the models in the extensive small open economy literature, such as Galí and Monacelli (2005), which typically feature a sharp distinction between a fully flexible-price traded good (or foreign good) and a sticky-price non-traded good (or domestic good). ${ }^{7}$ The second case is a less heterogeneous economy with two sticky-price sectors. We calibrate this economy to match roughly the micro evidence by Bils and Klenow (2004) and assess the quantitative relevance of our theoretical results.

\footnotetext{
${ }^{6}$ For a discussion of the early contributions of this literature, some of which are based on the dichotomous assumption of sticky prices and flexible prices, see Canzoneri et al. (2003). For a recent application of models with this dichotomous assumption to the analysis of the oil and food price shock, see Bodenstein et al. (2008) and Anand and Prasad (2010).

${ }^{7}$ See for instance Catao and Chang (2010), for a recent application of this framework to the analysis of large swings in world food prices and their implications for monetary policy.
} 
Of particular interest is the result that by having some weight on the expected flexible-price inflation, a rule may lead to a non-learnable fundamental-driven equilibrium. The intuition is straightforward in the dichotomous economy, where the expected flexible-price inflation is negatively correlated to current sticky-price output. As a result, a greater weight on flexible-price inflation makes the rule respond more negatively to current sticky-price output. This response, however, goes against the policy prescriptions of "leaning against the wind" by Taylor (1999a)-nominal interest rates should be adjusted positively, and more than one-for-one, in response to inflation, and positively to current output. In the two-sector model, expected deviations of the sticky-price inflation from the REE may be reinforced by a rule that responds positively to expected flexible-price inflation, and therefore negatively to current sticky-price output. This is because this rule may bring about a decrease in the real interest rate which adds to sticky-price inflationary pressures, pushing the economy away from a targeted equilibrium. So the equilibrium may become non-learnable.

Our work distinguishes itself from the New-Keynesian literature on interest rate rules and macroeconomic instability by focusing on the inflation measure as the potential source of instability. Benhabib et al. (2001), Carlstrom and Fuerst (2001), Clarida et al. (2000), and Woodford (2003), among others, discuss extensively how the rule's degree of aggressiveness towards inflation (i.e., active versus passive rules) and the timing of inflation (i.e., expected, contemporaneous or past inflation) may affect the extent to which rules induce endogenous fluctuations driven by self-fulfilling expectations. Bullard and Mitra (2002), Eusepi (2007), Evans and Honkapohja (2003), Evans and McGough (2005), Llosa and Tuesta (2008), and Preston (2006), among others, investigate the importance of similar features in the context of learning. But despite their seminal contributions, all these works do not consider the measure of inflation as a central bank policy choice that may affect both the learning and determinacy of equilibrium.

Our work also distinguishes from Carlstrom et al. (2006a), which is an important exception in the literature. By studying rules that respond to contemporaneous inflation, Carlstrom et al. (2006a) claim that the measure of inflation is irrelevant to ensure equilibrium determinacy, as long as the Taylor principle holds. Our work goes beyond theirs in two aspects. First, it tests the robustness of their claim by analyzing forward-looking and backward-looking rules. Second, it adds learnability as an extra criterion to select the inflation measure. Both aspects are crucial for revealing that, for a degree of heterogeneous price stickiness consistent with micro data, the inflation measure seems to be relevant for equilibrium determinacy and learnability. In this regard, our results suggest that the Taylor principle does not necessarily apply either at the sectorial level or at the aggregate level.

Finally by using different selection criteria, our work differs from the optimal monetary policy literature that selects the measure of inflation based on welfare analysis. Aoki (2001), Benigno (2004) and Mankiw and Reis (2003) recommend to target the inflation with the higher degree of price stickiness from an optimal targeting rule perspective. Our work supports a similar conclusion from an instrumental rule perspective (Taylor rules) and under the different criteria of equilibrium determinacy and learnability. The recent works by Anand and Prasad (2010), Bodenstein et al. (2008), and Catao and Chang (2010) study the implications of large swings in world food and oil price swings for the inflation measure of instrumental rules. Here again the selection criteria is welfare-based, abstracting from the fact that the inflation measure might exacerbate macroeconomic volatility by inducing 
non-fundamental-driven equilibria and unstable learning dynamics.

The remainder of this paper is organized as follows. Section II describes the general two-sector NewKeynesian economy. Section III presents the main results by pursuing the equilibrium determinacy and learnability analyses for forward-looking, contemporaneous, and backward-looking rules that respond to different measures of inflation. Section IV tests and discusses the robustness of our results under some extensions of our framework, including an explicit response to output in the rule and a different timing of real money balances in utility. Finally, Section V concludes.

\section{The Model}

In this section we develop a New-Keynesian dynamic stochastic general equilibrium model of a closed economy with two goods. ${ }^{8}$ The economy is populated by a continuum of infinitively-lived household-firm units, each of whom derives utility from consuming, not working, and the liquidity services of money. ${ }^{9}$ The unit consumes two types of composite goods - which we call $F$ and $S$ and are made of a continuum of intermediate differentiated products, indexed by $j \in[0,1]$. The unit also produces the $j$-th variety of the composite good $F$ (from now on, good $j$ of type $F$ ) and the $j$-th variety of the composite good $S$ (from now on, good $j$ of type $S$ ). That is, each production unit is split in two sub-units, specializing in a type- $F$ and type- $S$ good, respectively. We will often refer to these two sub-units as sector $F$ and sector $S$.

\section{A. The Household-Firm Unit}

Each household's intertemporal preferences are described by:

$$
\mathbb{E}_{0} \sum_{t=0}^{\infty} \beta^{t}\left\{\frac{c_{t}^{1-\sigma}}{1-\sigma}-A\left[\frac{\left(h_{t}^{s F}\right)^{1+\varphi^{F}}}{1+\varphi^{F}}+\frac{\left(h_{t}^{s S}\right)^{1+\varphi^{S}}}{1+\varphi^{S}}\right]+\frac{\left(m_{t}\right)^{1+\varphi^{m}}}{1+\varphi^{m}}\right\}
$$

where $\mathbb{E}_{0}$ denotes the (potentially) subjective expectations operator conditional on information available at time $0, c_{t}$ is aggregate consumption, $h_{t}^{s F}\left(h_{t}^{s S}\right)$ denotes the labor effort supplied to produce $\operatorname{good} F(S)$, and $m_{t}=\frac{M_{t}}{P_{t}}$ are the real money balances in terms of the Consumer Price Index (CPI) $P_{t}$ to be defined below. In addition, $\beta \in(0,1)$ is the subjective discount factor, $\sigma>0$ is the constant relative risk aversion coefficient, and $\varphi^{i}$ corresponds to the inverse of the Frisch elasticity of the labor supplied to sector $i$, for $i=F, S .^{10}$

\footnotetext{
${ }^{8}$ To be more realistic, one should consider a more general $N$-goods economy, for $N$ arbitrarily large. However, this would just enlarge the set of policy options (namely, the inflation measure to include in the policy rule) available to the policy-maker, without adding any further insight to the focus of our discussion. Hence, without loss of generality, we let $N=2$.

${ }^{9}$ Our results would equally hold in a model with separate consumption and production decisions, with firms operating in two distinct productive sectors.

${ }^{10}$ From the point of view of the household, the labor supplied to different sectors are not perfect substitutes, as in Woodford (2003). Clearly, this specification nests the RBC indivisible labour case, which occurs for $\varphi^{F}=\varphi^{S}=0$.
} 

$c_{t}^{S}$

Aggregate consumption is defined by the CES aggregator of the two consumption goods $c_{t}^{F}$ and

$$
c_{t}=\left[\eta^{\frac{1}{\xi}}\left(c_{t}^{F}\right)^{\frac{\xi-1}{\xi}}+(1-\eta)^{\frac{1}{\xi}}\left(c_{t}^{S}\right)^{\frac{\xi-1}{\xi}}\right]^{\frac{\xi}{\xi-1}}
$$

where $\xi$ is the intratemporal elasticity of substitution between goods, while $\eta$ is a parameter associated with the share of good $F$ in aggregate consumption. ${ }^{11}$

In this set-up, good $F$ and good $S$ can be Edgeworth complements, neutral, or substitutes, depending on the intertemporal and intratemporal elasticities of substitution $\frac{1}{\sigma}$ and $\xi$. To see this, define the utility of aggregate consumption as $U=\frac{c_{t}^{1-\sigma}}{1-\sigma}$. Then by (2) the sign of $\frac{\partial^{2} U}{\partial c^{F} \partial c^{S}}$ is determined by the sign of $\frac{1}{\sigma}-\xi$. Therefore, the goods are complements when $\xi<\frac{1}{\sigma}$, neutral if $\xi=\frac{1}{\sigma}$, and substitutes when $\xi>\frac{1}{\sigma}$. Furthermore, under the specification in (2), the CPI index corresponds to

$$
P_{t}=\left[\eta\left(P_{t}^{F}\right)^{1-\xi}+(1-\eta)\left(P_{t}^{S}\right)^{1-\xi}\right]^{\frac{1}{1-\xi}}
$$

and the relative demands for both goods are given by

$$
c_{t}^{F}=\eta\left(\frac{P_{t}^{F}}{P_{t}}\right)^{-\xi} c_{t} \quad \text { and } \quad c_{t}^{S}=(1-\eta)\left(\frac{P_{t}^{S}}{P_{t}}\right)^{-\xi} c_{t},
$$

satisfying $P_{t} c_{t}=P_{t}^{F} c_{t}^{F}+P_{t}^{S} c_{t}^{S}$.

The relative price $q_{t}$ is defined as $P_{t}^{F} / P_{t}^{S}$ and satisfies the law of motion given by:

$$
q_{t}=q_{t-1}\left(\frac{\pi_{t}^{F}}{\pi_{t}^{S}}\right)
$$

where $\pi_{t}^{i}=P_{t}^{i} / P_{t-1}^{i}$ denotes the "gross" inflation for good $i=F, S$.

The two goods are made of a continuum of differentiated intermediate products and defined as

$$
c_{t}^{F}=\left[\int_{0}^{1}\left(c_{t}^{F j}\right)^{\frac{\mu^{F}-1}{\mu^{F}}} d j\right]^{\frac{\mu^{F}}{\mu^{F}-1}} \text { and } c_{t}^{S}=\left[\int_{0}^{1}\left(c_{t}^{S j}\right)^{\frac{\mu^{S}-1}{\mu^{S}}} d j\right]^{\frac{\mu^{S}}{\mu^{S}-1}},
$$

with $\mu^{F}>1$ (respectively $\mu^{S}>1$ ) denoting the intratemporal elasticity of substitution between any pair of goods of $c_{t}^{F}$ (respectively $c_{t}^{S}$ ). The relative demands of the good $j$ of type $F$ and the good $j$ of type $S$ are then given by

$$
c_{t}^{F j}=\left(\frac{P_{t}^{F j}}{P_{t}^{F}}\right)^{-\mu^{F}} c_{t}^{F} \quad \text { and } \quad c_{t}^{S j}=\left(\frac{P_{t}^{S j}}{P_{t}^{S}}\right)^{-\mu^{S}} c_{t}^{S},
$$

\footnotetext{
${ }^{11}$ Our results do not depend on the above particular functional forms. They still hold for utility functions that satisfy $U\left(c_{t}^{F}, c_{t}^{S}\right)+H\left(h_{t}^{s F}\right)+L\left(h_{t}^{s S}\right)+J\left(m_{t}\right)$ and the following: a) $U(),. H(),. L($.$) and J($.$) are continuous and twice differentiable;$ b) $U($.$) , is strictly increasing \left(U_{F} \equiv \frac{\partial U}{\partial c_{t}^{F}}>0, U_{S}>0\right)$ and strictly concave $\left(U_{F F}<0, U_{S S}<0\right)$, and $U_{F S}=U_{S F}$, $U_{S S}-U_{S F} \frac{U_{S}}{U_{F}}<0$ and $U_{F F} U_{S S}-\left(U_{F S}\right)^{2}>0$; c) $H($.$) and L($.$) are strictly decreasing \left(H_{h} \equiv \frac{d H}{d h_{t}^{s F}}<0, L_{h} \equiv \frac{\partial L}{\partial h_{t}^{s S}}<0\right)$ and concave $\left(H_{h h} \leq 0, L_{h h} \leq 0\right)$; and d) $J($.$) is strictly increasing \left(J_{m}>0\right)$ and strictly concave $\left(J_{m m}<0\right)$. Details can be provided from the authors upon request.
} 
for any $j \in[0,1]$.

The representative unit produces the intermediate goods $j$ of type $F$ and type $S$ using simple linear production technologies:

$$
y_{t}^{F j}=z_{t} h_{t}^{F j} \quad \text { and } \quad y_{t}^{S j}=z_{t} h_{t}^{S j}
$$

with $h_{t}^{F j}$ and $h_{t}^{S j}$ denoting the labor hired. Both productions occur in a regime of monopolistic competition. We assume labor to be perfectly mobile within each sector, implying that the nominal wages are equal within sectors, i.e., $W_{t}^{F j}=W_{t}^{F}$ and $W_{t}^{S j}=W_{t}^{S}$ for any $j \in[0,1]$. However, labor is completely immobile across sectors and, therefore, $W_{t}^{F}$ and $W_{t}^{S}$ can differ in equilibrium. ${ }^{12}$ The economy-wide aggregate technology shock $z_{t}$ is the only source of fundamental intrinsic uncertainty and behaves as: ${ }^{13}$

$$
\hat{z}_{t}=\psi \hat{z}_{t-1}+\nu_{t}
$$

where $\hat{z}_{t}=\ln \left(z_{t}\right), \psi \in(0,1)$, and $\nu_{t} \sim \operatorname{Normal}\left(0, \varrho^{2}\right)$.

The monopolist unit sets the prices $P_{t}^{F j}$ and $P_{t}^{S j}$ of the variety $j$ of both intermediate goods it supplies, taking as given their respective aggregate demands $\left(\frac{P_{t}^{F j}}{P_{t}^{F}}\right)^{-\mu^{F}} C_{t}^{F}$ and $\left(\frac{P_{t}^{S j}}{P_{t}^{S}}\right)^{-\mu^{S}} C_{t}^{S}$, where $C_{t}^{F}$ and $C_{t}^{S}$ denote the economy-wide demand levels for the composite goods $F$ and $S$. Hence, output is demand-determined in both sectors:

$$
z_{t} h_{t}^{F j} \geq C_{t}^{F}\left(\frac{P_{t}^{F j}}{P_{t}^{F}}\right)^{-\mu^{F}} \quad \text { and } \quad z_{t} h_{t}^{S j} \geq C_{t}^{S}\left(\frac{P_{t}^{S j}}{P_{t}^{S}}\right)^{-\mu^{S}} \quad \text { for any } j \in[0,1]
$$

We introduce nominal price rigidities as in Rotemberg (1982). When setting the price of a good $j$ of type $i$, the unit is subject to a quadratic resource cost given by $\frac{\gamma^{i}}{2}\left(\frac{P_{t}^{i j}}{P_{t-1}^{i j}}-\bar{\pi}^{i}\right)^{2} P_{t}^{i} C_{t}^{i}$ for $i=F, S$, with the adjustment cost coefficient $\gamma^{i}$ measuring the degree of nominal rigidity in sector $i$ : the higher $\gamma^{i}$ the larger the degree of price stickiness in that sector. The difference between $\gamma^{F}$ and $\gamma^{S}$ will capture the different degree of price stickiness across sectors. Without loss of generality, we assume that $\gamma^{F}<\gamma^{S}$, i.e., in sector $F$ prices are always relatively more flexible, but not necessarily completely flexible, than in sector $S$. But note that our set-up embeds the case of fully flexible prices and perfect competition in sector $F$, by setting $\gamma^{F}=0$ and $\mu^{F} \rightarrow+\infty$.

Besides money $M_{t}$, the household-firm unit has access to a government bond $B_{t}$, which pays a nominal interest rate $R_{t}-1$. The unit also pays taxes $T_{t}^{g}$ and receives labor subsidies from the

\footnotetext{
${ }^{12}$ This assumption is quite common in the related literature (see Carlstrom et al., 2006a,b, Carvalho, 2006, Bouakez et al., 2009, Catao and Chang, 2010). It can be motivated by the existence of agent-specific skills, firm-specific inputs, costs of learning new jobs, or any other real friction that makes job reallocation costly. Results for the case of perfect labor mobility are available from the authors upon request.

${ }^{13}$ Since we will be focusing on equilibrium determinacy and E-stability the assumption of an aggregate technology shock rather than two sector-specific shocks is without loss of generality. It simplifies the notation without affecting the results of the paper.
} 
government at the rates $v^{F}$ and $v^{S} .{ }^{14}$ The budget constraint can then be written as follows:

$$
\begin{aligned}
M_{t}+B_{t}+P_{t} c_{t}+P_{t} T_{t}^{g} \leq & R_{t-1} B_{t-1}+M_{t-1}+\left(1+v^{F}\right) W_{t}^{F} h_{t}^{s F}+\left(1+v^{S}\right) W_{t}^{S} h_{t}^{s S} \\
& +\left[P_{t}^{F j} z_{t} h_{t}^{F j}-W_{t}^{F} h_{t}^{F j}-\frac{\gamma^{F}}{2}\left(\frac{P_{t}^{F j}}{P_{t-1}^{F j}}-\bar{\pi}^{F}\right)^{2} P_{t}^{F} C_{t}^{F}\right] \\
+ & {\left[P_{t}^{S j} z_{t} h_{t}^{S j}-W_{t}^{S} h_{t}^{S j}-\frac{\gamma^{S}}{2}\left(\frac{P_{t}^{S j}}{P_{t-1}^{S j}}-\bar{\pi}^{S}\right)^{2} P_{t}^{S} C_{t}^{S}\right], }
\end{aligned}
$$

where the right-hand side represents the sources of the unit's wealth, including interest payments on government bonds, money holdings, labor wage income, and net profits from the production of both goods. This wealth is used to finance new money holdings, purchase government bonds, and pay for total consumption and taxes, as described by the left-hand side of the constraint.

The representative household-firm unit chooses the set of stochastic processes $\left\{c_{t}, c_{t}^{F}, c_{t}^{S}, h_{t}^{s F}, h_{t}^{s S}\right.$, $\left.h_{t}^{F j}, h_{t}^{S j}, P_{t}^{F j}, P_{t}^{S j}, M_{t}, B_{t}\right\}_{t=0}^{\infty}$ in order to maximize (1) subject to (7), (8) and a standard Non-Ponzi game condition on total assets, given initial holdings $\left(B_{-1}, M_{-1}\right)$, and the set of stochastic processes $\left\{R_{t}, P_{t}, P_{t}^{F}, P_{t}^{S}, W_{t}^{F}, W_{t}^{S}, T_{t}^{g}, C_{t}^{F}, C_{t}^{S}, z_{t}\right\}_{t=0}^{\infty}$. The relevant first order conditions are:

$$
\begin{gathered}
c_{t}^{-\sigma}=\beta R_{t} \mathbb{E}_{t}\left(\frac{c_{t+1}^{-\sigma}}{\pi_{t+1}}\right), \\
A\left(h_{t}^{s i}\right)^{\varphi^{i}} c_{t}^{\sigma}=\left(1+v^{i}\right) \frac{W_{t}^{i}}{P_{t}}, \text { for } i=F, S,
\end{gathered}
$$

and

$$
\begin{aligned}
& c_{t}^{-\sigma} C_{t}^{i}\left\{\left(\frac{P_{t}^{i j}}{P_{t}^{i}}\right)^{-\mu^{i}}\left[\mu^{i}\left(1-\frac{M C_{t}^{i}}{P_{t}^{i j}}\right)-1\right]+\gamma^{i}\left(\frac{P_{t}^{i j}}{P_{t-1}^{i j}}-\bar{\pi}^{i}\right) \frac{P_{t}^{i}}{P_{t-1}^{i j}}\right\} \\
= & \beta \mathbb{E}_{t}\left[\frac{c_{t+1}^{-\sigma}}{\pi_{t+1}} P_{t+1}^{i} C_{t+1}^{i} \gamma^{i}\left(\frac{P_{t+1}^{i j}}{P_{t}^{i j}}-\bar{\pi}^{i}\right) \frac{P_{t+1}^{i j}}{\left(P_{t}^{i j}\right)^{2}}\right], \text { for } i=F, S,
\end{aligned}
$$

where $\pi_{t+1} \equiv P_{t+1} / P_{t}$ is the gross CPI inflation and $M C_{t}^{i} \equiv \frac{W_{t}^{i}}{z_{t}}$ is the nominal marginal cost of producing good $j$ of type $i$.

The interpretation of these equations is straightforward. Condition (9) is the standard Euler equation for consumption $c_{t}$. Equation (10) makes the marginal rate of substitution between labor allocated to the production of the type $i$ good and consumption equal to the respective real subsidized wage. Equation (11) is the optimal price setting condition for a unit producing good $j$ of type $i$. It simply states that, in every period, the unit sets a new price $P_{t}^{i j}$ taking into account its impact on current profits, as reflected by the terms on the left-hand side of (11), and the impact on future profits, as captured by the term on the right-hand side of the same equation.

\footnotetext{
${ }^{14}$ The introduction of constant subsidies $v^{F}$ and $v^{S}$ simplifies the steady-state analysis and allows us to derive analytical results. See the Appendix C.
} 
While the first order conditions (9)-(11) together with the appropriate transversality condition are necessary and sufficient if $\mathbb{E}_{t}$ is assumed to be the expectations operator conditional on all available information (hence, rational), the same might not apply for more general settings. In this paper, learning takes the form of the "Euler Equation" approach by Evans and Honkapohja (2001), which remains the most common in the adaptive learning literature related to our work. More specifically, it is assumed that economic agents are boundedly-rational and choose based on the first order conditions (9)-(11), which describe the relevant marginal benefits and marginal costs associated with each economic decision.

We focus on a symmetric equilibrium in which the monopolistic units belonging to the same sector pick the same price. That is, $P_{t}^{F j}=P_{t}^{F}$ and $P_{t}^{S j}=P_{t}^{S}$ for any $j \in[0,1]$. Given that they face the same wage rates $W_{t}^{S}$ and $W_{t}^{F}$, and the same technology, they also demand the same amounts of labor, $h_{t}^{F j}=h_{t}^{F}$ and $h_{t}^{S j}=h_{t}^{S}$ for any $j \in[0,1]$. Combining the first order conditions of this maximization problem with the clearing conditions in the labor markets $\left(h_{t}^{s F}=h_{t}^{F}\right.$ and $\left.h_{t}^{s S}=h_{t}^{S}\right)$ and in the goods markets $\left(c_{t}^{S}=C_{t}^{S}=z_{t} h_{t}^{S}-\frac{\gamma^{S}}{2}\left(\pi_{t}^{S}-\bar{\pi}^{S}\right)^{2} C_{t}^{S}\right.$ and $\left.c_{t}^{F}=C_{t}^{F}=z_{t} h_{t}^{F}-\frac{\gamma^{F}}{2}\left(\pi_{t}^{F}-\bar{\pi}^{F}\right)^{2} C_{t}^{F}\right)$, we obtain the New-Keynesian Phillips curves (NKPC):

$$
\gamma^{i}\left(\pi_{t}^{i}-\bar{\pi}^{i}\right) \pi_{t}^{i}=\gamma^{i} \beta \mathbb{E}_{t}\left[\mathcal{H}_{t, t+1}^{i}\left(\pi_{t+1}^{i}-\bar{\pi}^{i}\right) \pi_{t+1}^{i}\right]+\mu^{i}\left(m c_{t}^{i}-\frac{\mu^{i}-1}{\mu^{i}}\right) \text {, for } i=F, S,
$$

where $\pi_{t}^{i} \equiv \frac{P_{t}^{i}}{P_{t-1}^{i}}, \mathcal{H}_{t, t+1}^{i} \equiv\left(\frac{c_{t+1}}{c_{t}}\right)^{-\sigma}\left(\frac{C_{t+1}^{i}}{C_{t}^{i}}\right)\left(\frac{\pi_{t+1}^{i}}{\pi}\right)$ and $m c_{t}^{i} \equiv \frac{W_{t}^{i}}{z_{t} P_{t}^{i}}$.

\section{B. The Government}

The government issues two nominal liabilities (money and a domestic bond), provides labor income subsidies, pays interest on its debt and receives revenues from seigniorage and lump-sum taxes. It follows a generic Ricardian fiscal policy, by setting taxes in order to satisfy its intertemporal budget constraint and a Non-Ponzi game condition.

Following Taylor (1993), we define monetary policy as an interest rate feedback rule. The government sets the gross nominal interest rate $R_{t}$ as an increasing and continuous function of the deviation of a chosen measure of inflation $\pi_{t}^{o}$, with respect to a target $\bar{\pi}^{o}$. Formally,

$$
R_{t}=\bar{R}\left[\mathbb{E}_{t}\left(\frac{\pi_{t+k}^{o}}{\bar{\pi}^{o}}\right)\right]^{\phi_{\pi}} \quad \text { where } \quad k=-1,0,1, \quad \phi_{\pi}>1
$$

$\bar{R}$ is the interest rate target and $\mathbb{E}$ is the expectation operator. For simplicity, we also assume that the targets $\bar{\pi}^{o}$ and $\bar{R}$ correspond to the steady-state levels of $\pi_{t}^{o}$ and $R_{t}$.

Some remarks are in order regarding the specification in (13). First, we focus on rules that satisfy the Taylor principle, also known as active rules. These are rules that respond to increases in inflation with a more than one-for-one increase in the interest rate, i.e., $\phi_{\pi}>1$. And second, we allow for different policy rule timings, including a forward-looking rule that responds to the deviation

of the expected future inflation from the target, $\mathbb{E}_{t}\left(\frac{\pi_{t+1}^{o}}{\bar{\pi}^{o}}\right)$; a contemporaneous rule that reacts to 
the current inflation deviation $\left(\frac{\pi_{t}^{o}}{\bar{\pi}^{o}}\right)$; or a backward-looking rule that responds to the past inflation deviation $\left(\frac{\pi_{t-1}^{o}}{\bar{\pi}^{o}}\right)$.

To underscore the role of the inflation measure of the rule as a policy variable, we define the inflation measure in the rule (13) as:

$$
\pi_{t}^{o}=\omega \pi_{t}^{F}+(1-\omega) \pi_{t}^{S},
$$

where in principle the government can freely pick the weight $\omega$. This set-up is general enough to nest the cases of a rule responding to the most flexible-price inflation only $(\omega=1)$, the stickiest price (core) inflation $(\omega=0)$, or any arbitrary measure of headline inflation $(\omega \in(0,1))$, including the CPI inflation case of $\omega=\eta$.

\section{Equilibrium Determinacy and Stability under Learning}

Given $\phi_{\pi}>1$, the objective of our analysis is to assess under what conditions on the policy parameter $\omega$, the economy displays a determinate (unique) and expectationally stable (E-stable) Rational Expectations Equilibrium (REE). Both equilibrium determinacy and E-stability are particularly desirable from the point of view of the policymaker. Under determinacy, the equilibrium dynamics are solely driven by fundamentals, ruling out the effects of extrinsic uncertainty such as noise, market sentiment and all other factors often referred to as "sunspots." Under E-stability, even if boundedly rational, economic agents will eventually learn the fundamental REE by recursive least squares regressions on past data, and the economy will converge to the targeted equilibrium.

To derive our main results we consider two different price-setting cases embedded in our previously discussed set-up. First, we study an economy with the strong dichotomy of a fully flexible-price sector and a sticky-price sector. This case allows us to derive analytical results and, as mentioned in the Introduction, reflects the popular two-sector price setting assumption in both the optimal monetary policy literature in closed economies and the small open economy literature. Furthermore, we believe this case captures the essential message of the micro and macro evidence, namely: goods differ greatly in how frequently their prices change with some of them changing prices every quarter or more often. From a modeling perspective, this implies a non-zero measure of fully flexible-price sectors, especially if the model is calibrated to match quarterly data. The second case attempts to take the micro evidence more seriously and therefore calibrates the main set-up, with price-stickiness in both sectors, to the evidence provided by Bils and Klenow (2004). This helps us show that our main analytical results hold (numerically) for a general two-sector economy with a lower, but still realistic, degree of heterogeneous price stickiness across sectors.

\section{A. The Methodology}

We now provide a brief description of our methodology for the equilibrium determinacy and learning analyses. We derive, in particular, the E-stability conditions that a representation of an equilibrium 
needs to satisfy in order to be learnable.

The characterization of a Rational Expectation Equilibrium (REE) follows Blanchard and Kahn (1980). For each of the aforementioned rules we log-linearize and write the model as

$$
E_{t} \widehat{x}_{t+1}=J \widehat{x}_{t}+\Lambda \widehat{z}_{t}
$$

where $E$ is now the rational expectations operator, $\widehat{x}_{t}$ is a $s \times 1$ vector of endogenous variables, $\widehat{z}_{t}$ is the technology shock, and $J$ and $\Lambda$ are conformable matrices. The characterization depends on the number of roots of $J$ that lie outside the unit circle: if this number is equal to the number of non-predetermined variables, there is a unique equilibrium (determinacy); when this number is less than the number of non-predetermined variables, then there are multiple equilibria (indeterminacy); and if this number of roots is greater than the number of non-predetermined variables, then almost surely there exists no non-explosive solution to the model.

The learning analysis draws on Evans and Honkapohja (2001). Agents no longer are endowed with rational expectations but, instead, have adaptive rules to form expectations using recursive least squares updating. We focus, in particular, on E-stability as a learning criterion: a representation of an equilibrium is learnable if it is E-stable. The reason is that under E-stability, recursive leastsquares learning is in general locally convergent to the REE. This is shown analytically by Marcet and Sargent (1989) and Evans and Honkapohja (2001) for models with a unique equilibrium and, through simulations, by Evans and McGough (2005) for a New-Keynesian model that displays "sunspot" equilibria.

We proceed to derive the conditions under which a REE is E-stable. To do so, we reduce the model associated with each of the interest rate rules to:

$$
\widehat{x}_{t}=\Upsilon+\Omega \mathbb{E}_{t} \widehat{x}_{t+1}+\Gamma \widehat{x}_{t-1}+\Pi \widehat{z}_{t} \quad \text { and } \quad \widehat{z}_{t}=\psi \widehat{z}_{t-1}+\nu_{t},
$$

where $\widehat{x}_{t}$ is a $s \times 1$ vector of endogenous variables, $\widehat{z}_{t}$ is the exogenous fundamental shock. $\psi \in(0,1)$ and $\nu_{t} \backsim \operatorname{Normal}\left(0, \varrho^{2}\right)$. In addition, $\Upsilon, \Omega, \Gamma$, and $\Pi$ are conformable matrices of constants, while $\mathbb{E}_{t}$ denotes in general (non-rational) expectations.

Consider first the E-stability conditions for a fundamental-driven REE. In this case we focus on the learnability of McCallum's (1983) Minimal State Variable (MSV) solution. Therefore we assume agents follow a Perceived Law of Motion (PLM) $\widehat{x}_{t}=\mathcal{A}+\mathcal{F} \widehat{x}_{t-1}+\mathcal{N} \widehat{z}_{t}$, where $\mathcal{A}$, $\mathcal{F}$, and $\mathcal{N}$ are conformable matrices. Iterating forward the PLM and using it to eliminate all the forecasts in the model, we obtain the implied Actual Law of Motion (ALM) $\widehat{x}_{t}=\mathfrak{B}\left[\Upsilon+\Omega \mathcal{A}+\Gamma \widehat{x}_{t-1}+(\Omega \mathcal{N} \psi+\Pi) \widehat{z}_{t}\right]$ with $\mathfrak{B}=(I-\Omega \mathcal{F})^{-1}$. The PLM and ALM, in turn, define the T-mapping $T(\mathcal{A}, \mathcal{F}, \mathcal{N})=\left(\mathcal{A}^{A}, \mathcal{F}^{A}, \mathcal{N}^{A}\right)$, where $\mathcal{A}^{A}=\mathfrak{B}(\Upsilon+\Omega \mathcal{A}), \mathcal{F}^{A}=\mathfrak{B} \Gamma$, and $\mathcal{N}^{A}=\mathfrak{B}(\Omega \mathcal{N} \psi+\Pi) .{ }^{15}$ The fixed points of this mapping are

\footnotetext{
${ }^{15} \mathrm{~A}$ fundamental part in the learnability analysis consists of making explicit what agents know when they form their forecasts. In the E-stability analysis literature, it is common to assume that when agents form their expectations $\mathbb{E}_{t} \widehat{x}_{t}$, they do not know $\widehat{x}_{t}$. This may be, however, inconsistent with the assumptions that we used to derive the first order conditions of the household-firm unit problem. For instance, recall that in that derivation $\mathbb{E}_{t} P_{t}^{S j}=P_{t}^{S j}$. Therefore to be consistent, in the analysis to follow we assume that when forming expectations $\mathbb{E}_{t} \widehat{x}_{t}$ agents know $\widehat{x}_{t}$.
} 
the REE. Following Evans and Honkapohja (2001), we say that the MSV representation of a REE is E-stable if all the eigenvalues of the matrices of the derivatives

$$
D T_{\mathcal{A}}=(I-\Omega \mathcal{F})^{-1} \Omega, D T_{\mathcal{F}}=\left[(I-\Omega \mathcal{F})^{-1} \Gamma\right]^{\prime} \otimes\left[(I-\Omega \mathcal{F})^{-1} \Omega\right], \text { and } D T_{\mathcal{N}}=\psi \otimes\left[(I-\Omega \mathcal{F})^{-1} \Omega\right]
$$

evaluated at the REE fixed point, have real parts less than one. On the contrary, the MSV solution is not E-stable if any of the eigenvalues has a real part larger than one.

To derive the E-stability conditions for stationary "sunspot" equilibria, we concentrate on Common Factor (CF) representations, as in Evans and McGough (2005). When $\widehat{x}_{t}$ is a vector of nonpredetermined variables, the PLM for the CF representation corresponds to $\widehat{x}_{t}=\mathcal{A}+\mathcal{N} \widehat{z}_{t}+\mathcal{G} \varepsilon_{t}$, where $\mathcal{A}, \mathcal{N}$, and $\mathcal{G}$ are conformable vectors. $\varepsilon_{t}$, in turn, is a "sunspot" following $\varepsilon_{t}=v \varepsilon_{t-1}+\varpi_{t}$, where $|v|<1$ and $\varpi_{t}$ is an arbitrary martingale difference sequence. Then T-mapping associated with this PLM corresponds to $T(\mathcal{A}, \mathcal{N}, \mathcal{G})=\left(\mathcal{A}^{A}, \mathcal{N}^{A}, \mathcal{G}^{A}\right)$, where $\mathcal{A}^{A}=(\Upsilon+\Omega \mathcal{A}), \mathcal{N}^{A}=(\Omega \mathcal{N} \psi+\Pi)$, and $\mathcal{G}^{A}=\Omega \mathcal{G} v$. Using this mapping, the E-stability conditions can be stated as having all the eigenvalues of the matrices

$$
D T_{\mathcal{A}}=\Omega, \quad D T_{\mathcal{N}}=\psi \otimes \Omega, \quad \text { and } \quad D T_{\mathcal{G}}=v \otimes \Omega,
$$

with real parts less than one.

\section{B. The Strong Dichotomy: A Flexible-Price and Sticky-Price Economy}

This section provides the results of the case in which sector $F$ features perfect competition $\left(\mu^{F} \rightarrow+\infty\right)$ and fully flexible prices $\left(\gamma^{F}=0\right)$, while sector $S$ keeps the standard New-Keynesian characteristics. ${ }^{16}$ For reasons of space, we present analytical results only for forward-looking rules. That is, for $k=1$ in equation (13). Our interest for rules responding to expectations is motivated by the empirical estimates in Clarida et al. (2000), and more recently in Cogley and Sargent (2005) and Boivin (2006). ${ }^{17}$

Since $\gamma^{F}=0$ and $\mu^{F} \rightarrow+\infty$, then equation (12) for $i=F$ reduces to $m c_{t}^{F}=1$. The rest of the equations of our set-up still hold. Without loss of generality, we focus on the case where the steady-state relative price $\bar{q}$ is equal to one, and hence where $\bar{\pi}^{F}=\bar{\pi}^{S}=\bar{\pi}^{18}$ We then proceed by log-linearizing the equations of the model around the steady state of the economy. Log-linearized

\footnotetext{
${ }^{16}$ The assumption of perfect competition helps us simplify the notation. Our results would equally hold for any finite value $\mu^{F}$.

${ }^{17}$ Analytical results for the case of a contemporaneous interest rate rule, as well as numerical results for the case of a backward-looking interest rate rule are available from the authors upon request.

${ }^{18}$ This is not unusual in the analysis of two-sector models. See for instance Galí and Monacelli (2005). As shown in the Appendix A, the introduction of labor subsidies allows us to focus on the steady state where $\bar{q}=1$, simplifying the analysis. This is without loss of generality. Moreover, with $\bar{q}=1$, it is possible to show that at the steady state: $\bar{R}=\frac{\bar{\pi}}{\beta}, \bar{\pi}^{F}=\bar{\pi}^{S}=\bar{\pi}^{o}=\bar{\pi}$,

$$
\bar{c}^{S}=\left[\frac{\left(\mu^{S}-1\right)(1-\eta)^{\sigma}}{\mu^{S} A}\right]^{\frac{1}{\varphi^{S}+\sigma}}, \quad \bar{c}=\frac{\bar{c}^{S}}{1-\eta}, \quad \bar{c}^{F}=\eta \bar{c}, \quad \bar{h}^{S}=\bar{c}^{S}, \quad \text { and } \quad \bar{h}^{F}=\bar{c}^{F}=\eta \bar{c}
$$
}


variables will be denoted with a hat. For instance, log-linearized sticky-price consumption will be denoted as $\widehat{c}_{t}^{S}$, where $\widehat{c}_{t}^{S}=\log \left(\frac{c_{t}^{S}}{\bar{c}^{S}}\right)$ and $\bar{c}^{S}$ corresponds to its steady-state level.

Under a forward-looking rule, log-linearizing (13) and (14) yields:

$$
\widehat{r}_{t}=\phi_{\pi} \mathbb{E}_{t} \widehat{\pi}_{t+1}^{o}
$$

with

$$
\widehat{\pi}_{t+1}^{o}=\omega \widehat{\pi}_{t+1}^{F}+(1-\omega) \widehat{\pi}_{t+1}^{S} .
$$

On the demand side, by combining the (log-linearized) expressions for (5), the CES specification in (2), the CPI (3), equation (10), $m c_{t}^{F}=1$, the definition of the relative price $q_{t}$, and the market clearing condition $c_{t}^{F}=z_{t} h_{t}^{F}$, we obtain the following relationship between the flexible-price and sticky-price consumptions:

$$
\widehat{c}_{t}^{F}=\varsigma \widehat{c}_{t}^{S}+\varkappa_{1} \widehat{z}_{t}
$$

where $\varsigma \equiv \frac{(1-\eta)(1-\xi \sigma)}{\left(\varphi^{F}+\sigma \eta\right) \xi+1-\eta}$ and $\varkappa_{1} \equiv \frac{\left(1+\varphi^{F}\right) \xi}{\left(\varphi^{F}+\sigma \eta\right) \xi+1-\eta}$. Note that $\operatorname{sign}(\varsigma)=\operatorname{sign}(1-\xi \sigma)$. Hence, along any equilibrium path, the two consumptions are positively (negatively) related when the goods are Edgeworth complements (substitutes) i.e. $\xi<\frac{1}{\sigma}\left(\xi>\frac{1}{\sigma}\right)$. When the goods are neutral $\left(\xi=\frac{1}{\sigma}\right.$, hence $\varsigma=0$ ), flexible-price consumption depends only on the aggregate technology shock.

Combining the log-linearized versions of the demand functions in (4) with (5) and (21), we find that:

$$
\widehat{c}_{t}^{S}-\widehat{c}_{t-1}^{S}=\frac{1}{\theta}\left(\widehat{\pi}_{t}^{F}-\widehat{\pi}_{t}^{S}\right)+\frac{\varkappa_{1}}{\theta \xi}\left(\widehat{z}_{t}-\widehat{z}_{t-1}\right)
$$

where $\theta \equiv \frac{\sigma+\varphi^{F}}{\left(\varphi^{F}+\sigma \eta\right) \xi+1-\eta}>0$. This equation captures, in part, the expenditure switching or substitution effect that will be crucial to understand our results: for a given sticky-price inflation, the growth rate of sticky-price consumption is positively related to flexible-price inflation.

The demand side characterization is completed by log-linearizing the Euler equation (9) to obtain:

$$
\widehat{c}_{t}=\mathbb{E}_{t} \widehat{c}_{t+1}-\frac{1}{\sigma}\left(\widehat{r}_{t}-\mathbb{E}_{t} \widehat{\pi}_{t+1}\right)
$$

with the (log) CPI inflation given by

$$
\widehat{\pi}_{t+1}=\eta \widehat{\pi}_{t+1}^{F}+(1-\eta) \widehat{\pi}_{t+1}^{S} .
$$

Using this and the log-linearized versions of (2) and (3), with equations (21) and (22) allows us to derive a log-linearized Euler equation for sticky-price consumption:

$$
\widehat{c}_{t}^{S}-\mathbb{E}_{t} \widehat{c}_{t+1}^{S}=-\alpha\left(\widehat{r}_{t}-\mathbb{E}_{t} \widehat{\pi}_{t+1}^{S}\right)-\varkappa_{2} \widehat{z}_{t},
$$

with $\alpha \equiv[\sigma(\eta \varsigma+1-\eta)+\eta \theta]^{-1}>0$ and $\varkappa_{2} \equiv(1-\psi) \varkappa_{1} \eta\left(\alpha \frac{\sigma \xi-1}{\xi}\right)$.

The supply side, on the other hand, is described by the log-linearized version of the NKPC (12):

$$
\widehat{\pi}_{t}^{S}=\beta \mathbb{E}_{t} \widehat{\pi}_{t+1}^{S}+\kappa \widehat{m c} \widehat{c}_{t}^{S},
$$


with $\kappa \equiv \frac{\left(\mu^{S}-1\right)}{\gamma^{S}\left(\bar{\pi}^{S}\right)^{2}}>0$, which relates the current inflation of the sticky-price good $\widehat{\pi}_{t}^{S}$ to the inflation expectations $\mathbb{E}_{t} \widehat{\pi}_{t+1}^{S}$ and the marginal cost of producing this good $\widehat{m c}_{t}^{S}$. Furthermore, by combining the log-linearized versions of (2), (3), (10) for $i=S$, and $c_{t}^{S}=z_{t} h_{t}^{S}-\frac{\gamma^{S}}{2}\left(\pi_{t}^{S}-\bar{\pi}\right)^{2} c_{t}^{S}$ with equations (5) and (21), we can express the real marginal costs in terms of log-linearized sticky-price consumption:

$$
\widehat{m c}_{t}^{S}=\vartheta \widehat{c}_{t}^{S}+\mathfrak{h} \widehat{z}_{t}
$$

where $\vartheta \equiv \varphi^{S}+\frac{1}{\alpha}>0$ and $\mathfrak{h} \equiv-\left(1+\varphi^{S}\right)-\varkappa_{1} \eta\left(\frac{1-\sigma \xi}{\xi}\right)$. Replacing this into (26) yields:

$$
\widehat{\pi}_{t}^{S}=\beta \mathbb{E}_{t} \widehat{\pi}_{t+1}^{S}+\delta \widehat{c}_{t}^{S}+\mathfrak{s} \widehat{z}_{t},
$$

where $\delta \equiv \kappa \vartheta>0$ and $\mathfrak{s} \equiv \kappa \mathfrak{h}<0$, which clearly shows that, in the model, an excess of demand for the sticky-price good is translated into sticky-price inflationary pressures.

Based on this log-linearized set of equations, we can provide the following definition of a fundamentaldriven equilibrium in this economy.

Definition 1 Given the stochastic process $\left\{\widehat{z}_{t}\right\}_{t=0}^{\infty}$, an equilibrium is a set of stochastic processes $\left\{\widehat{r}_{t}\right.$, $\left.\widehat{\pi}_{t}^{o}, \widehat{\pi}_{t}^{F}, \widehat{\pi}_{t}^{S}, \widehat{c}_{t}^{S}\right\}_{t=0}^{\infty}$ satisfying a) the rule (19); b) the definition of full inflation (20); c) equation (22); d) the Euler equation for sticky-price consumption (25); and e) the New-Keynesian Phillips curve (28). 19

Some Analytical Results. Proposition 1 provides the results for the equilibrium determinacy analysis. It states that conditions under which an active forward-looking rule delivers a locally unique REE crucially depend on the weight of the flexible-price inflation $\omega$ in the inflation measure $\widehat{\pi}_{t}^{o}$, and the types of goods considered (complements, neutral, or substitutes). It reveals that across these types, as the weight on the flexible-price inflation increases, the rule is more prone to induce multiple equilibria, even if the Taylor Principle holds (i.e., $\phi_{\pi}>1$ ).

Proposition 1 Let $\phi_{1}(\omega) \equiv \frac{\delta \alpha+2(1+\beta)}{\delta \alpha+2(1+\beta) \tau \omega}>0$ and $\omega^{*}=\frac{1}{\tau}$, where $\tau \equiv \alpha \theta$. Consider the forward-looking interest rate in (19)-(20) with $\phi_{\pi}>1$, and assume that agents have Rational Expectations, i.e., $\mathbb{E}=E$.

a) For any $\omega \in(0,1)$ when $\xi \geq \frac{1}{\sigma}$ (substitutes or neutral), or any $\omega \in\left(0, \omega^{*}\right)$ when $\xi<\frac{1}{\sigma}$ (complements), the following hold: 1) there exists a unique local REE if $1<\phi_{\pi}<\phi_{1}(\omega)$, and 2) there exist multiple local REE if $\phi_{\pi}>\phi_{1}(\omega)$.

b) For any $\omega \in\left(\omega^{*}, 1\right)$ when $\xi<\frac{1}{\sigma}$ (complements), there exist multiple local REE for any $\phi_{\pi}>1$.

Proof. See the Appendix B.

\footnotetext{
${ }^{19}$ Note that once we determine the stochastic processes $\left\{\widehat{r}_{t}, \widehat{\pi}_{t}^{o}, \widehat{\pi}_{t}^{F}, \widehat{\pi}_{t}^{S}, \widehat{c}_{t}^{S}\right\}_{t=0}^{\infty}$, we can pin down the rest of the stochastic processes by using the previously discussed equations that are excluded from the definition. For instance, given $\left\{\widehat{c}_{t}^{S}\right\}_{t=0}^{\infty}$ we can use equation $(21)$ to derive $\left\{\widehat{c}_{t}^{F}\right\}_{t=0}^{\infty}$.
} 
The existence of multiple equilibria allows for the presence of stationary "sunspot" equilibria. This, in turn, raises the question of whether there are representations of these equilibria that are learnable. If this were the case, then economic agents would be more likely to coordinate their actions and push the economy towards these equilibria driven by "sunspots." The following proposition shows that, in fact, there are learnable Common Factor $(\mathrm{CF})$ representations, whose learnability clearly depends on the weight of the flexible-price inflation $\omega$ in the headline inflation $\widehat{\pi}_{t}^{o}$.

Proposition 2 Let $\phi_{1}(\omega) \equiv \frac{\delta \alpha+2(1+\beta)}{\delta \alpha+2(1+\beta) \tau \omega}>0, \phi_{2}(\omega) \equiv \frac{1}{\tau \omega}>0$ and $\omega^{*}=\frac{1}{\tau}$. Consider the forwardlooking rule in (19)-(20) with $\phi_{\pi}>1$. For any $\omega \in(0,1)$, when $\frac{1}{\sigma} \leq \xi$ (substitutes or neutral), or any $\omega \in\left(0, \omega^{*}\right)$, when $\frac{1}{\sigma}>\xi$ (complements), the CF representation of stationary "sunspot" equilibria is learnable in the E-stability sense if $\phi_{1}(\omega)<\phi_{\pi}<\phi_{2}(\omega)$.

Proof. See the Appendix B.

But even if one focuses on fundamental-driven equilibria, ignoring the multiple REE problem, the weight on the flexible-price inflation $\omega$ and the types of goods play key roles for the presence of unstable learning dynamics. The next proposition presents the results for the learnability of the MSV representation of fundamental-driven equilibria (from now on MSV-REE). It shows that, given the response coefficient $\phi_{\pi}$, putting higher weights $\omega$ makes a rule more prone to induce fundamental-driven equilibria that are not learnable in the E-stability sense. ${ }^{20}$

Proposition 3 Let $\phi_{2}(\omega) \equiv \frac{1}{\tau \omega}>0$ and $\omega^{*}=\frac{1}{\tau}$. Consider the forward-looking interest rate rule in (19)-(20) with $\phi_{\pi}>1$, and concentrate on fundamental equilibria.

a) For any $\omega \in(0,1)$ when $\xi \geq \frac{1}{\sigma}$ (substitutes or neutral), or any $\omega \in\left(0, \omega^{*}\right)$ when $\xi<\frac{1}{\sigma}$ (complements), the MSV-REE is 1) learnable in the E-stability sense if $1<\phi_{\pi}<\phi_{2}(\omega)$, and 2) not learnable in the E-stability sense if $\phi_{\pi}>\phi_{2}(\omega)$.

b) For any $\omega \in\left(\omega^{*}, 1\right)$ when $\xi<\frac{1}{\sigma}$ (complements), the MSV-REE is not learnable in the E-stability sense for any $\phi_{\pi}>1$, .

Proof. See the Appendix B.

Figure 1 puts together the results from Propositions 1, 2, and 3. It plots, for each type of goods, the combinations of policy parameters $\omega$ and $\phi_{\pi}$ that deliver equilibrium determinacy or indeterminacy. It also shows the combinations for E-stable (learnable) or E-unstable (non-learnable) MSV-REE, as well as the combinations for E-stable CF representations of "sunspot" equilibria.

\footnotetext{
${ }^{20}$ The importance of learnability for the MSV solution is discussed in the recent debate on the meaningfulness of policy lessons from New-Keynesian models between Cochrane (2009) and McCallum (2009).
} 
Forward-Looking Rules
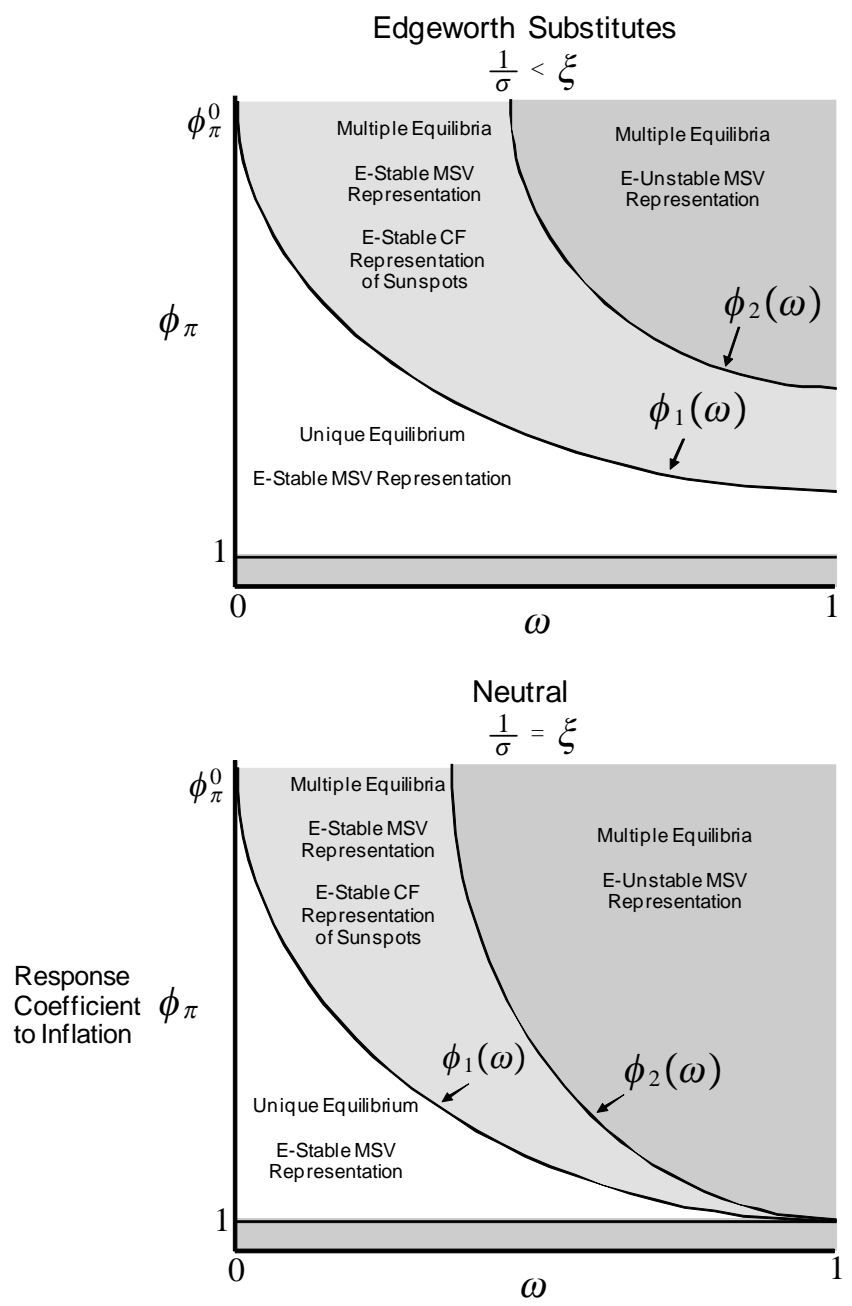

Edgeworth Complements

$\frac{1}{\sigma}>\xi$

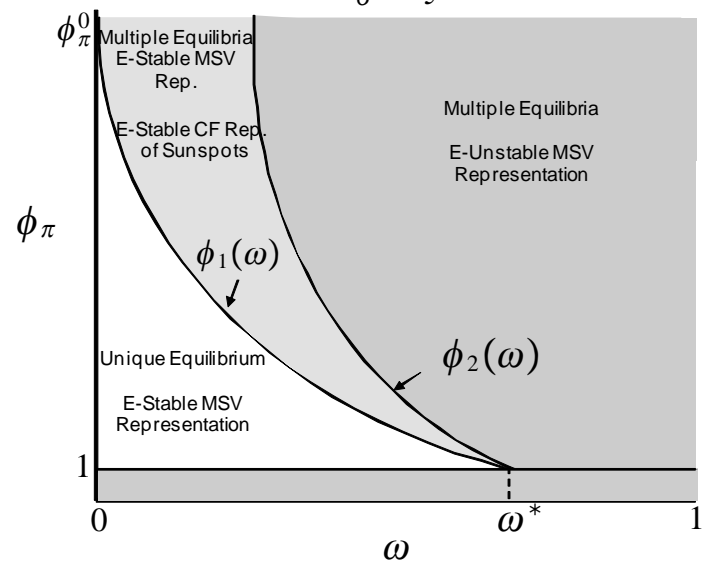

Weight on the Flexible-Price Inflation

Figure 1: Regions of determinacy and expectational stability for forward-looking rules, when varying the response coefficient to inflation $\phi_{\pi}$ and the weight on expected flexible-price inflation $\omega$ in the headline inflation. 
In this figure, the one-sector New-Keynesian model analyzed in Bullard and Mitra (2002) corresponds to the vertical axis, where $\omega=0$, meaning that the rule responds solely to expected sticky-price inflation. In this case, and regardless of the goods type, equilibrium determinacy requires the active rule to satisfy an upper threshold $\phi_{\pi}^{0} \equiv 1+\frac{2(1+\beta)}{\alpha \delta}$, so that $\phi_{\pi}<\phi_{\pi}^{0}$. According to Bullard and Mitra, this threshold takes very high values for realistic calibrations and, therefore, is non-binding for policy design. E-stability of the MSV solution, on the other hand, is ensured by any active rule $\left(\phi_{\pi}>1\right)$.

Some interesting results emerge for positive weights on the flexible-price inflation $(\omega>0)$, since the determinacy and E-stability bounds, $\phi_{1}(\omega)$ and $\phi_{2}(\omega)$, are decreasing in this weight.

First, across different types of goods, putting more weight on the flexible-price inflation shrinks the range $1<\phi_{\pi}<\phi_{1}(\omega)$ under which a rule delivers a unique and learnable equilibrium. When $\phi_{\pi}>\phi_{1}(\omega)$, the rule induces multiple equilibria and, from the learning perspective, gives rise to the following instability problems. If $\phi_{1}(\omega)<\phi_{\pi}<\phi_{2}(\omega)$, then the rule induces "sunspot" equilibria with learnable CF representations. While if $\phi_{\pi}>\phi_{2}(\omega)$ then the rule leads to fundamental equilibria with non-learnable MSV representations. This contrasts starkly with Bullard and Mitra's results for active forward-looking rules, which suggest that the funtamental MSV solution is always learnable, even under multiple equilibria.

Second, the determinacy conditions are generally more stringent that those related to the Estability of the MSV solution. That is, for any $\omega \in(0,1)$ when $\xi \geq \frac{1}{\sigma}$, and for any $\omega \in\left(0, \omega^{*}\right)$ when $\xi<\frac{1}{\sigma}$, we have that $\phi_{2}(\omega)>\phi_{1}(\omega)$, and therefore determinacy implies E-stability, but not viceversa.

Third, the lower the elasticity of substitution across goods, the more stringent the determinacy and E-stability conditions. In fact, simple algebra shows that, for any $\omega \in(0,1)$ when $\xi \geq \frac{1}{\sigma}$ (substitute or neutral goods), and for any $\omega \in\left(0, \omega^{*}\right)$ when $\xi<\frac{1}{\sigma}$ (complement goods), both frontiers $\phi_{1}(\omega)$ and $\phi_{2}(\omega)$ are increasing in the elasticity $\xi$.

And fourth, for complement goods, increasing $\omega$ can be even more harmful. For these goods there is a threshold $\omega^{*}$ beyond which any active rule leads to multiple equilibria and makes the MSV solution non-learnable. ${ }^{21}$

Some Intuition. We provide a simple intuition for our results in the context of complement goods, although a similar intuition can be derived for substitute or neutral goods. We analyze the adaptive learning process of economic agents following an initial deviation from the fundamental REE. Under adaptive learning, agents make forecasts using recursive least squares estimations on available data. Though we assume that they have the right functional form of the MSV solution, they might still overestimate or underestimate the impact of the technology shock on inflation and output. Then we explain how this initial deviation can be reinforced or offset by the policy rule.

\footnotetext{
${ }^{21}$ In the limiting case of $\omega=1$, when the rule responds solely to the flexible-price inflation, it can be proved that the range of $\phi_{\pi}$ for which there is a unique and learnable equilibrium is non-empty, only for substitute goods. For neutral and complement goods, the equilibrium is always indeterminate and the MSV solution is E-unstable for any $\phi_{\pi}>1$. The proof of this result relies on a continuity argument for $\omega$, following the lines of the proofs of Propositions 1 and 3.
} 
Key to develop our intuition is the following expression for the real interest rate:

$$
\widehat{r}_{t}-\mathbb{E}_{t} \widehat{\pi}_{t+1}^{S}=-\left(\frac{1-\phi_{\pi}}{1-\tau \omega \phi_{\pi}}\right) \mathbb{E}_{t} \widehat{\pi}_{t+1}^{S}, \text { with } \tau \equiv \alpha \theta,
$$

which is derived from the rule and equations (22) and (25). Under complement goods $\tau>1$, as shown by Lemma 1 in the Appendix B.

Assume that boundely-rational agents expect sticky-price inflation to be higher than in the REE. If the central bank sets $\omega \in\left(\frac{1}{\tau}, 1\right)$, then by (29) the real interest rate declines (with respect to the REE) for any $\phi_{\pi}>1$. This stimulates sticky-price consumption, by (25), which in turn induces higher stickyprice inflation, by (28). Over time, this mechanism leads to upward revisions of both the expected sticky-price inflation and consumption. As a result, the rule does not correct the initial deviation from the REE and, instead, moves the economy further away from it. This unstable dynamics is not ruled out if the central bank sets $\omega \in\left[0, \frac{1}{\tau}\right)$ and adopts an overly aggressive response to inflation, $\phi_{\pi}>\frac{1}{\tau \omega}$.

On the other hand, for $\omega \in\left[0, \frac{1}{\tau}\right)$ but $1<\phi_{\pi}<\frac{1}{\tau \omega}$, the initial deviation from the REE induces an increase in the real interest rate, thus slowing down consumption and putting downward pressure on sticky-price inflation. Agents would then correct downward their initial higher inflation forecast, which would move sticky-price consumption and the related inflation closer to the REE. By this self-correcting mechanism, agents will (eventually) make the economy converge to the fundamental REE.

Similarly, we could also construct a sunspot-driven equilibrium. However, in this case, the initial deviation from the fundamental REE would not be due to the agents' bounded rationality, but to the presence of non-fundamental belief shocks. Only under the conditions spelled in part a1) of Proposition 1, such initial deviation would not be self-fulfilled and agents would gradually revert to the fundamental REE.

To complement this intuition, we link our results to those policy recommendations in Bullard and Mitra (2002) and Taylor (1999a). They advocate for "leaning against the wind" policies: nominal interest rates should be adjusted positively, and more than one-for-one, in response to inflation, and positively to current output. In our model, a rule that puts some positive weight on expected flexibleprice inflation $(\omega>0)$ is actually equivalent to a rule that reacts negatively to current sticky-price output $\widehat{y}_{t}^{S}$. To see this, note that from equation (22) and $\widehat{y}_{t}^{S}=\widehat{c}_{t}^{S}$ the rule (19) can be written as: $\widehat{r}_{t}=\phi_{\pi} \mathbb{E}_{t} \widehat{\pi}_{t+1}^{S}+\phi_{y}\left(\mathbb{E}_{t} \widehat{y}_{t+1}^{S}-\widehat{y}_{t}^{S}\right)$ with $\phi_{y} \equiv \phi_{\pi} \omega \theta$. Then, for given $\phi_{\pi}$ and $\theta$, increasing the weight $\omega$ is equivalent to responding more aggressively to current sticky-price output $\widehat{y}_{t}^{S}$, but in a negative way. Clearly this negative response goes against the "leaning against the wind" policies. As a result, this response may bring about a decrease in the real interest rate, reinforcing potential sticky-price inflationary pressures and pushing the economy away from a targeted equilibrium.

\section{The General Set-up: The Bils-Klenow Calibration}

We focus now in the two-sector model with price rigidities in both sectors. From the log-linearization of the CPI index (3), the relative demands in (4), the relative price equation (5), the Euler Equation 
(9), the intratemporal conditions in (10) for $i=F, S$, the New-Keynesian Phillips curves in (12) for $i=F, S$, the rule specified in (13) and (14), as well as the definition $m c_{t}^{i}=\frac{W_{t}^{i}}{z_{t} P_{t}^{i}}$ for $i=F, S$, and the market clearing conditions, we obtain the following system:

$$
\begin{gathered}
\widehat{c}_{t}=\mathbb{E}_{t} \widehat{c}_{t+1}-\frac{1}{\sigma}\left[\widehat{r}_{t}-\eta \mathbb{E}_{t} \widehat{\pi}_{t+1}^{F}-(1-\eta) \mathbb{E}_{t} \widehat{\pi}_{t+1}^{S}\right], \\
\widehat{\pi}_{t}=\eta \widehat{\pi}_{t}^{F}+(1-\eta) \widehat{\pi}_{t}^{S} \\
\widehat{\pi}_{t}^{F}=\beta \mathbb{E}_{t} \widehat{\pi}_{t+1}^{F}+\kappa^{F}\left(\varphi^{F}+\sigma\right) \widehat{c}_{t}-\kappa^{F}\left(1+\xi \varphi^{F}\right)(1-\eta) \widehat{q}_{t}-\kappa^{F}\left(1+\varphi^{F}\right) \widehat{z}_{t}, \\
\widehat{\pi}_{t}^{S}=\beta \mathbb{E}_{t} \widehat{\pi}_{t+1}^{S}+\kappa^{S}\left(\varphi^{S}+\sigma\right) \widehat{c}_{t}+\kappa^{S}\left(1+\xi \varphi^{S}\right) \eta \widehat{q}_{t}-\kappa^{S}\left(1+\varphi^{S}\right) \widehat{z}_{t}, \\
\widehat{q}_{t}=\widehat{q}_{t-1}+\widehat{\pi}_{t}^{F}-\widehat{\pi}_{t}^{S},
\end{gathered}
$$

and

$$
\widehat{r}_{t}=\phi_{\pi} \omega \mathbb{E}_{t} \widehat{\pi}_{t+k}^{F}+\phi_{\pi}(1-\omega) \mathbb{E}_{t} \widehat{\pi}_{t+k}^{S},
$$

where $\widehat{z}_{t}$ follows $(6)$ and $\kappa^{i}=\frac{\mu^{i}-1}{\gamma^{i}(\bar{\pi})^{2}}$ for $i=F, S$. The system consists, respectively, of an aggregate Euler equation, the definition of CPI inflation, two New-Keynesian Phillips curves, the law of motion of the relative price, and the interest rate rule.

As analytical results are no longer attainable, we resort to the numerical analysis of a calibrated version of our economy. Most of our parameters are set in accordance to the existing literature. The parameter $A$ in (1) is picked such that in steady-state the household-firm unit allocates one third of its time to work. We assume equal steady-state gross inflation across sectors $\left(\bar{\pi}^{F}=\bar{\pi}^{S}=\bar{\pi}\right)$ and set the annual inflation equal to 3 percent. We pick $\mu^{F}=\mu^{S}=8$, implying a steady state gross mark-up of $15 \%$ in both sectors, while $\sigma=1$, i.e., a log-utility. Given $\sigma$, the cases of complement, susbtitute and neutral goods are captured by setting $\xi$ equal to $0.5,1.5$, and 1 , respectively. The remaining structural parameters are given the following values: $\beta=0.99, \psi=0.78$, and $\varphi^{F}=\varphi^{S}=2$ (a Frisch elasticity of labor supplied in both sectors equal to 0.5$).{ }^{22}$

\footnotetext{
${ }^{22}$ The assumptions of equal steady-state mark-ups and Frisch elasticities across sectors are not driving factors. We have experimented with various combinations of realistic values for $\varphi^{S} \neq \varphi^{F}$ and $\mu^{F} \neq \mu^{S}$, and found only mild quantitative changes in our results. More details are available from the authors upon request.
} 


\section{Table 1: Benchmark Calibration}

\begin{tabular}{cccccccccccc}
\hline \hline$\beta$ & $\sigma$ & $\eta$ & $A$ & $\varphi^{F}$ & $\varphi^{S}$ & $\mu^{F}$ & $\mu^{S}$ & $\gamma^{F}$ & $\gamma^{S}$ & $\bar{\pi}$ & $\psi$ \\
\hline \hline 0.99 & 1 & 0.4 & 19 & 2 & 2 & 8 & 8 & 0.67 & 74 & 1.0074 & 0.78 \\
\hline
\end{tabular}

The key aspect of our calibration are the price adjustment cost coefficients $\gamma^{F}$ and $\gamma^{S}$. We calibrate them using three key facts from the micro-evidence on price setting by Bils and Klenow (2004): the share of firms with an expected price duration of at most one quarter, i.e., the more flexible-price side of the economy; the average quarterly frequency of price change; and the average expected quarterly duration of price rigidity. ${ }^{23}$ As extensively described in the Appendix $\mathrm{C}$, we obtain $\eta=0.4, \gamma^{F}=0.67$ and $\gamma^{S}=74$. That is, our calibrated economy features a significant degree of sticky-price heterogeneity across the two sectors, with sector $F$ featuring very low adjustment costs to price changes. Since prices in sector $F$ are almost flexible, although not fully flexible, in the analysis to follow in this section we refer to the inflation of this sector as the flexible-price inflation. Table 1 summarizes our benchmark calibration.

\section{C.1. Forward-Looking Rules}

Figure 2 shows that, under a forward-looking rule, the determinacy and E-stability results for a realistic calibration of the two-sticky-price-sector economy are qualitatively similar to the analytical results of the "strong dichotomy" case in Subsection B (see Figure 1). Across different types of goods, putting a higher weight on the the flexible-price inflation restricts the set of forward-looking rules that induce a unique and learnable fundamental REE. Moreover, there exist combinations of $\phi_{\pi}$ and $\omega$ for which the economy displays learnable "sunspot" equilibria. In addition, when goods are complements, any active rule induces non-learnable fundamental-driven equilibria, if the weight $\omega$ is above a threshold $\omega^{*}$. With the strong dichotomy being a limiting case of the more general set-up and given the significant degree of sticky-price heterogeneity resulting from our calibration, the intuition provided above for the results should be unaltered.

To give a sense of the quantitative importance of our results, Table 2 quantifies the determinacy and E-stability thresholds for the case of Edgeworth complementarity.

In row a) we consider an active rule with a response to inflation $\phi_{\pi}=2$, which is the lower bound to the estimate of the "degree of activism" in the post-Volcker period by Cogley and Sargent (2005). We find that multiple equilibria arise for $\omega>0.44$, while the fundamental MSV-REE ceases to be learnable for $\omega>0.48$. This says that, even if the Taylor principle of $\phi_{\pi}>1$ holds, endogenous

\footnotetext{
${ }^{23}$ We refer to the Bils and Klenow (2004) evidence rather than to others, such as Nakamura and Steinsson (2008) or Klenow and Kryvtsov (2008), mainly because the data is readily available from their paper. Nevertheless, we are aware that, because of the inclusions of sales and forced substitutions, their reported statistics are somewhat upward-biased estimates of the true degree of price rigidity in the data.
} 


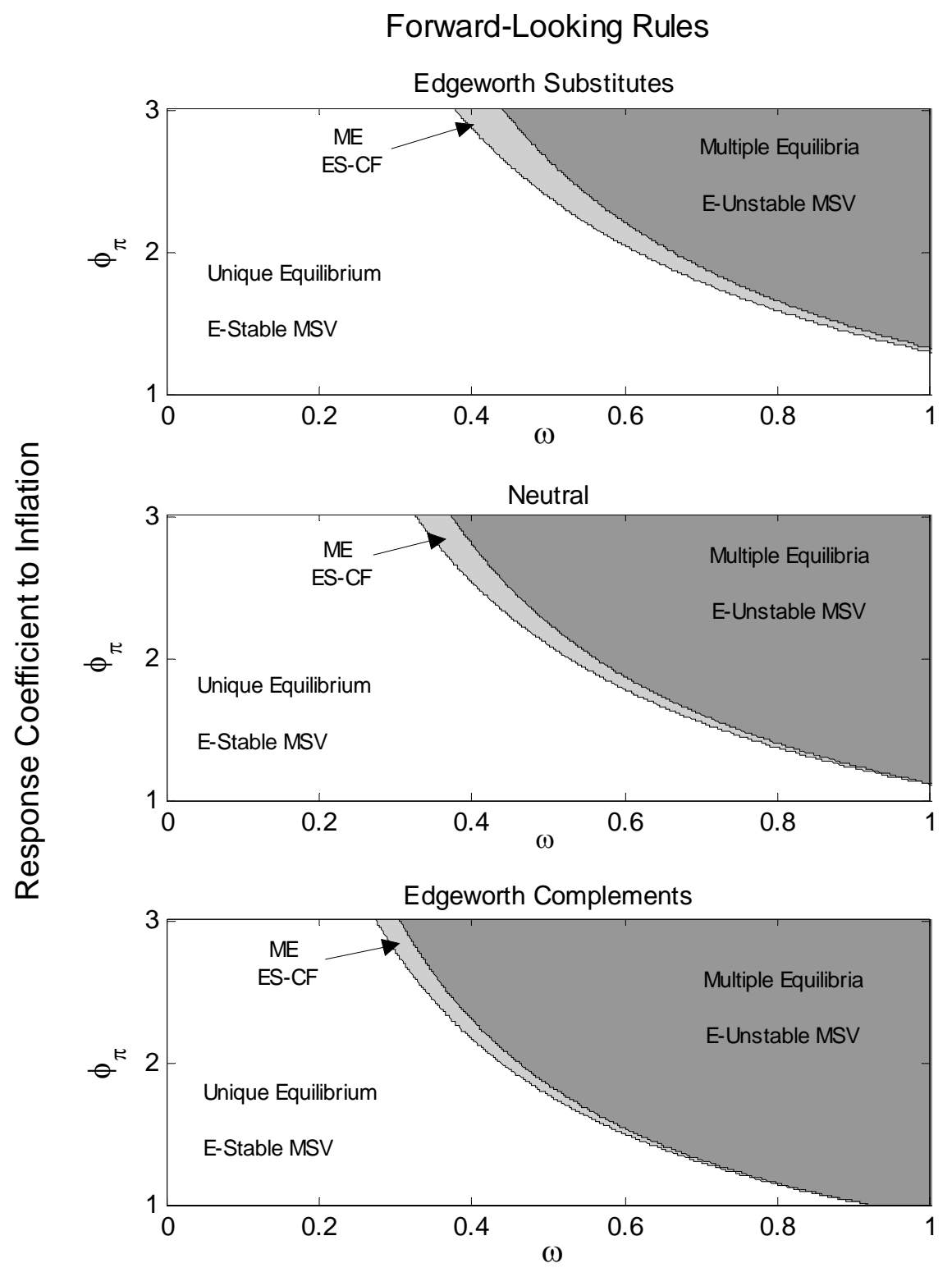

Weight on the Flexible-Price Inflation

Figure 2: Regions of determinacy and expectational stability for forward-looking rules. Parameters other than $\phi_{\pi}$ and $\omega$ are set at baseline values shown in Table 1.

Note: $\mathrm{ME}=$ Multiple Equilibria, ES-CF = E-Stable Common Factor representation of a "sunspot" equilibrium, and MSV = Minimum State Variable representation of a fundamental equilibrium. 
aggregate instability occurs for a weight on sector $F$ inflation just slightly above the CPI weight of $\eta=0.4$.

Row b) looks at the consequences of a positive $\omega$ from another angle. We fix its value below $\omega^{*}=0.92$. For instance, we set $\omega=\eta$ and identify the determinacy/E-stability bounds with respect to $\phi_{\pi}$. We obtain multiple equilibria for $\phi_{\pi}>2.18$ and a non-learnable MSV-REE for $\phi_{\pi}>2.3$. But for $\omega>0.92$ no active rule is stabilizing.

Table 2: Forward-Looking Rules

Determinacy and E-stability Bounds for Complement Goods

\begin{tabular}{l|c|c|c|c|c}
\hline \hline & \multicolumn{2}{|c|}{ Determinacy } & \multicolumn{3}{c}{ Learning } \\
\cline { 2 - 6 } & UE & ME & ES-MSV & ES-CF Sunspot & EU-MSV \\
\hline \hline a) $\phi_{\pi}=2$ & $0 \leq \omega<0.44$ & $0.44<\omega \leq 1$ & $0 \leq \omega<0.48$ & $0.44<\omega<0.48$ & $0.48<\omega \leq 1$ \\
b) $\omega=\eta=0.4$ & $1<\phi_{\pi}<2.18$ & $2.18<\phi_{\pi}$ & $1<\phi_{\pi}<2.3$ & $2.18<\phi_{\pi}<2.3$ & $2.3<\phi_{\pi}$ \\
\hline
\end{tabular}

Note: $\omega^{*}=0.92 . \mathrm{UE}=$ Unique equilibrim. $\mathrm{ME}=$ Multiple Equilibria.

$\mathrm{ES}-\mathrm{MSV}=\mathrm{E}$-stable (learnable) MSV representation of a fundamental equilibrium.

ES-CF Sunspot = E-stable (learnable) CF representation of a "sunspot" equilibrium.

$\mathrm{EU}-\mathrm{MSV}=\mathrm{E}$-unstable (non-learnable) MSV representation of a fundamental equilibrium.

\section{C.2. Other Timings for the Policy Rule}

Figure 3 displays the results for the case of the contemporaneous rule $\widehat{r}_{t}=\phi_{\pi} \widehat{\pi}_{t}^{o}$. In this case, and regardless of the types of goods (complements, substitutes, or neutral), the measure of inflation does not affect both equilibrium determinacy and E-stability. As long as the rule satisfies the Taylor principle of $\phi_{\pi}>1$, there is a unique and learnable equilibrium. This result is reminiscent of that by Carlstrom et al. (2006a), although they exclusively focus on determinacy, while here we complement their analysis by showing the learnability of the MSV representation. ${ }^{24}$

A different type of instability occurs under the backward-looking rule $\widehat{r}_{t}=\phi_{\pi} \widehat{\pi}_{t-1}^{o}$. Responding to past flexible-price inflation can induce explosive equilibria, that is, the economy diverges from the steady state equilibrium and give rise to unbounded fluctuations. Figure 4 shows that raising $\omega$ makes the backward-looking rule more prone to induce these equilibria, in particular for the case of Edgeworth complementarity. However, when the equilibrium is determinate, it is also learnable. ${ }^{25}$

\footnotetext{
${ }^{24}$ We find that this (numerical) result holds under any calibration considered. It can also be proved analytically for the dichotomous economy of Section B. There, $\widehat{r}_{t}=\phi_{\pi} \widehat{\pi}_{t}^{o}$ can be written as $\widehat{r}_{t}=\phi_{\pi} \widehat{\pi}_{t}^{S}+\phi_{\pi} \omega \theta\left(\widehat{c}_{t}^{S}-\widehat{c}_{t-1}^{S}\right)$. A positive $\omega$ is then equivalent to a positive response to sticky-price output growth, which is beneficial to determinacy.

${ }^{25}$ As in Bullard and Mitra (2002) we do not explore the E-stability of these explosive equilibria.
} 


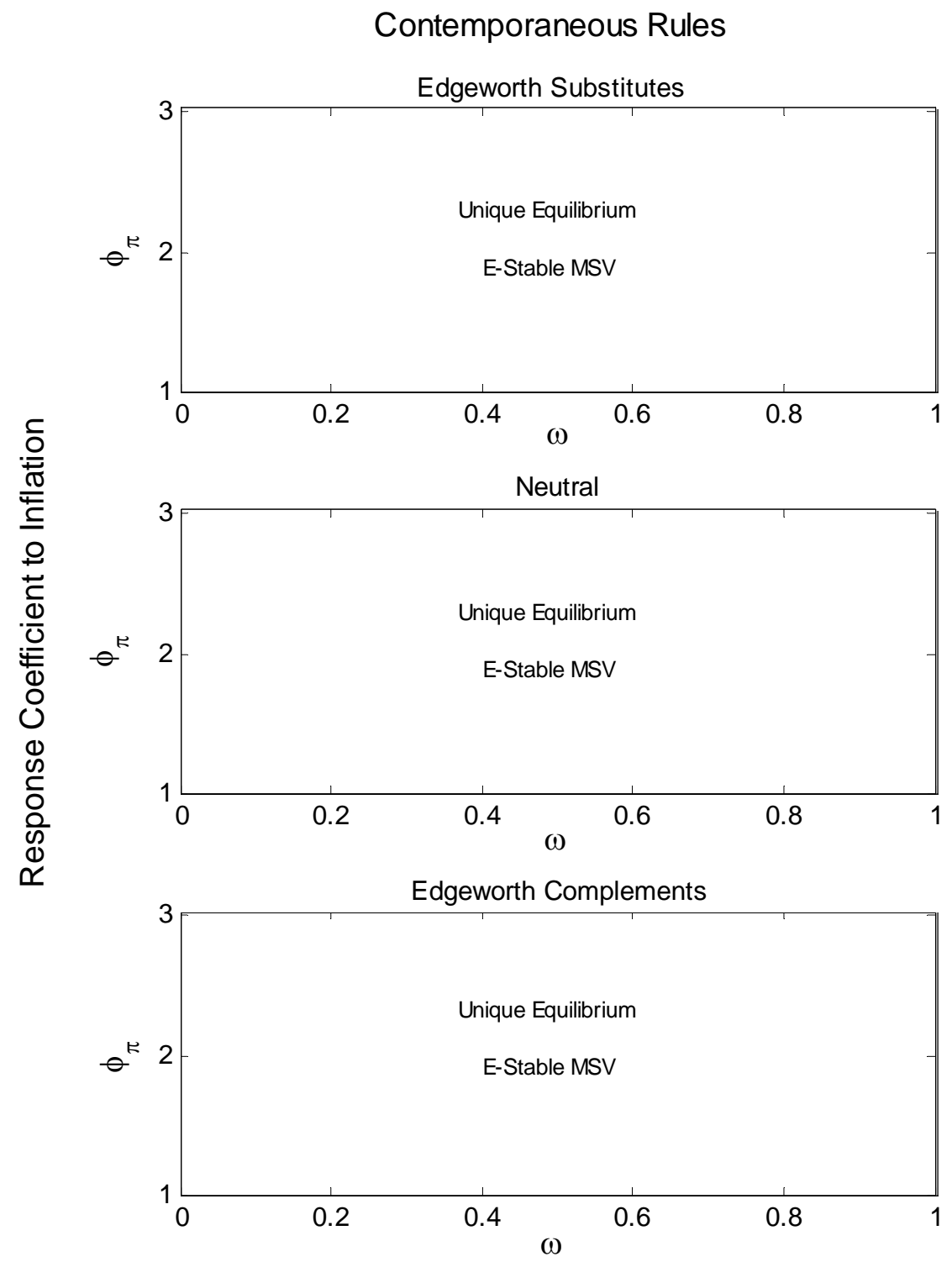

\section{Weight on the Flexible-Price Inflation}

Figure 3: Regions of determinacy and expectational stability for contemporaneous rules. Parameters other than $\phi_{\pi}$ and $\omega$ are set at baseline values shown in Table 1.

Note: $\mathrm{MSV}=$ Minimum State Variable representation of a fundamental equilibrium. 


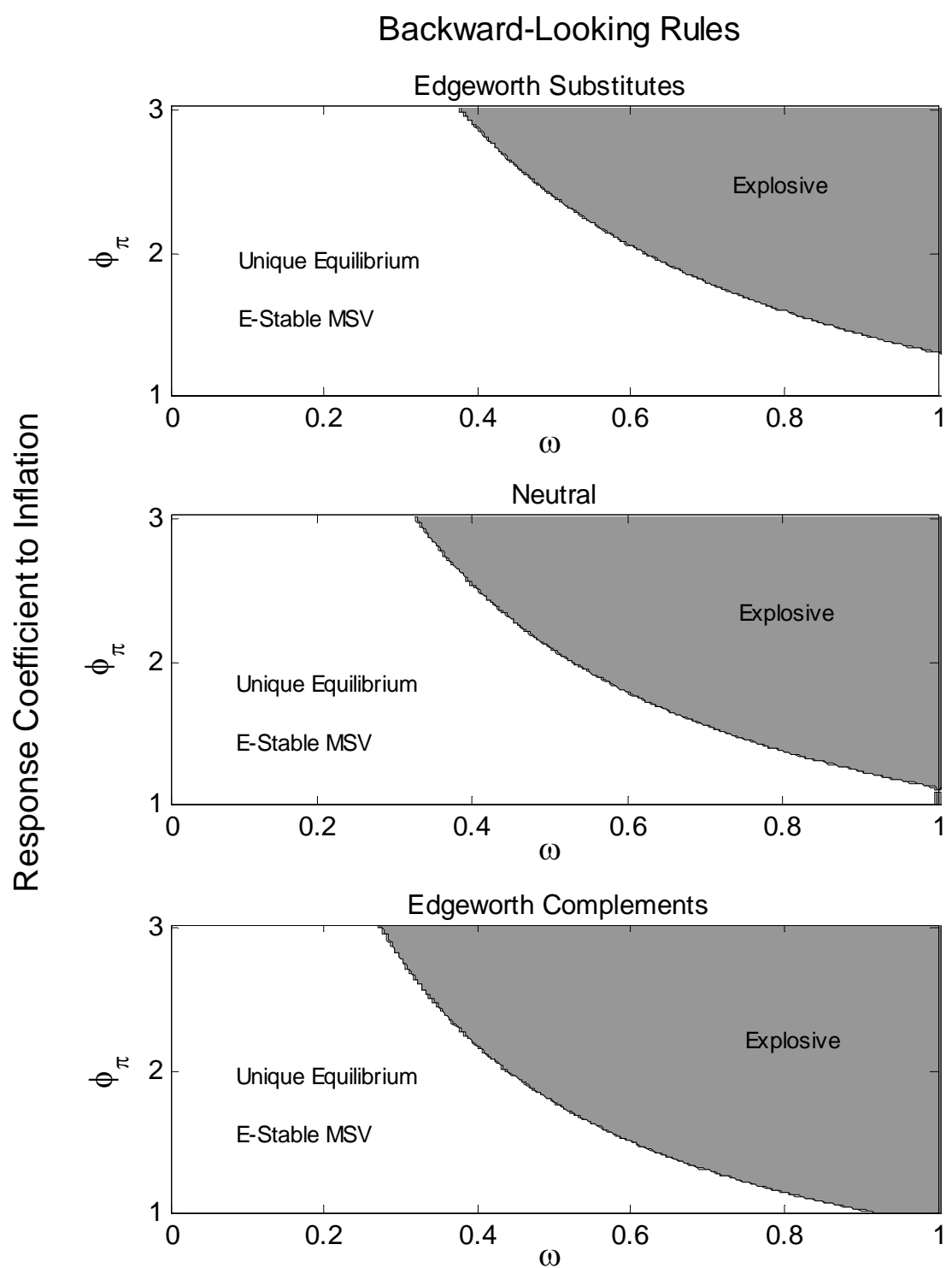

Weight on the Flexible-Price Inflation

Figure 4: Regions of determinacy and expectational stability for backward-looking rules. Parameters other than $\phi_{\pi}$ and $\omega$ are set at baseline values shown in Table 1.

Note: $\mathrm{MSV}=$ Minimum State Variable representation of a fundamental equilibrium. 
These results for other timings for the policy rule together with the results for forward-lookig rules suggest an important policy lesson to rule out potential sources of instability: to be on the safe side, policy makers should consider targeting the inflation of the sectors with a higher degree of price stickiness.

\section{C.3. Quantitative Sensitivity}

We conclude this Section by investigating the quantitative effect of varying the price stickiness heterogeneity across sectors as well as the share $\eta$ of flexible-price goods. Varying $\eta$ allows us to study the effects of responding to CPI inflation.

Aggregate Price Stickiness and Its Heterogeneity The variety of empirical estimates from the micro-based studies motivates us to assess how equilibrium determinacy and E-stability depend on different degrees of aggregate price rigidity and its heterogeneity across sectors. We focus on the average frequency of price change $f$ - a measure of aggregate price rigidity - and the relative standard deviation (also known as, the coefficient of variation) of the sectoral frequencies $f^{F}$ and $f^{S}$, which we take as a measure of the degree of heterogeneity and denote by $\Delta_{f} \in(0,1)$. More formally, we define

$$
f=\eta f^{F}+(1-\eta) f^{S} \quad \text { and } \quad \Delta_{f}=\frac{\sqrt{\eta\left(f^{F}-f\right)^{2}+(1-\eta)\left(f^{S}-f\right)^{2}}}{f}
$$

Concentrating on the frequencies of price change $\left(f^{i}\right.$ for $\left.i=F, S\right)$ rather than an average adjustment $\operatorname{costs}\left(\gamma^{i}\right.$ for $\left.i=F, S\right)$, is motivated by the fact that the available empirical evidence is about the frequency and not the cost of price changes. Clearly, this choice does not affect the interpretation of the results. There is a one to one mapping between these measures, as explained in the Appendix C. A higher $f^{i}$ implies a lower $\gamma^{i}$.

In this assessment, we consider $f \in[0.2,0.8]$, which includes the value $f=0.52$ used in our benchmark "Bils-Klenow" calibration. Then, for each possible pair $\left(f, \Delta_{f}\right)$, we set $f^{F}$ and $f^{S}$ such that they satisfy the equations in (35). Once $f^{F}$ and $f^{S}$ are identified, we compute the corresponding adjustment cost parameters $\gamma^{F}$ and $\gamma^{S}$ following the same calibration procedure explained in the Appendix C. We consider a forward-looking rule and an economy of equally-sized sectors, i.e., $\eta=0.5$, and set $\omega=\eta \cdot{ }^{26}$ All remaining parameters are as in Table 1. For reasons of space, we only present the results for the case of Edgeworth complementarity, i.e., $\xi=0.5$.

Several interesting results emerge (see Figure 5). First of all, for a given average frequency of price change just above 0.45 , endogenous aggregate instability arises when sticky-price heterogeneity is sufficiently high. Second, this upper threshold on heterogeneity is strictly decreasing in the aggregate level of rigidity: the more flexible prices are on average (i.e., the higher is $f$ ) the lower is the minimum

\footnotetext{
${ }^{26}$ Under this parametrization the only difference between the two sectors is the degree of price rigidity. Other than that, they are identical and treated symmetrically by the policy-maker.
} 


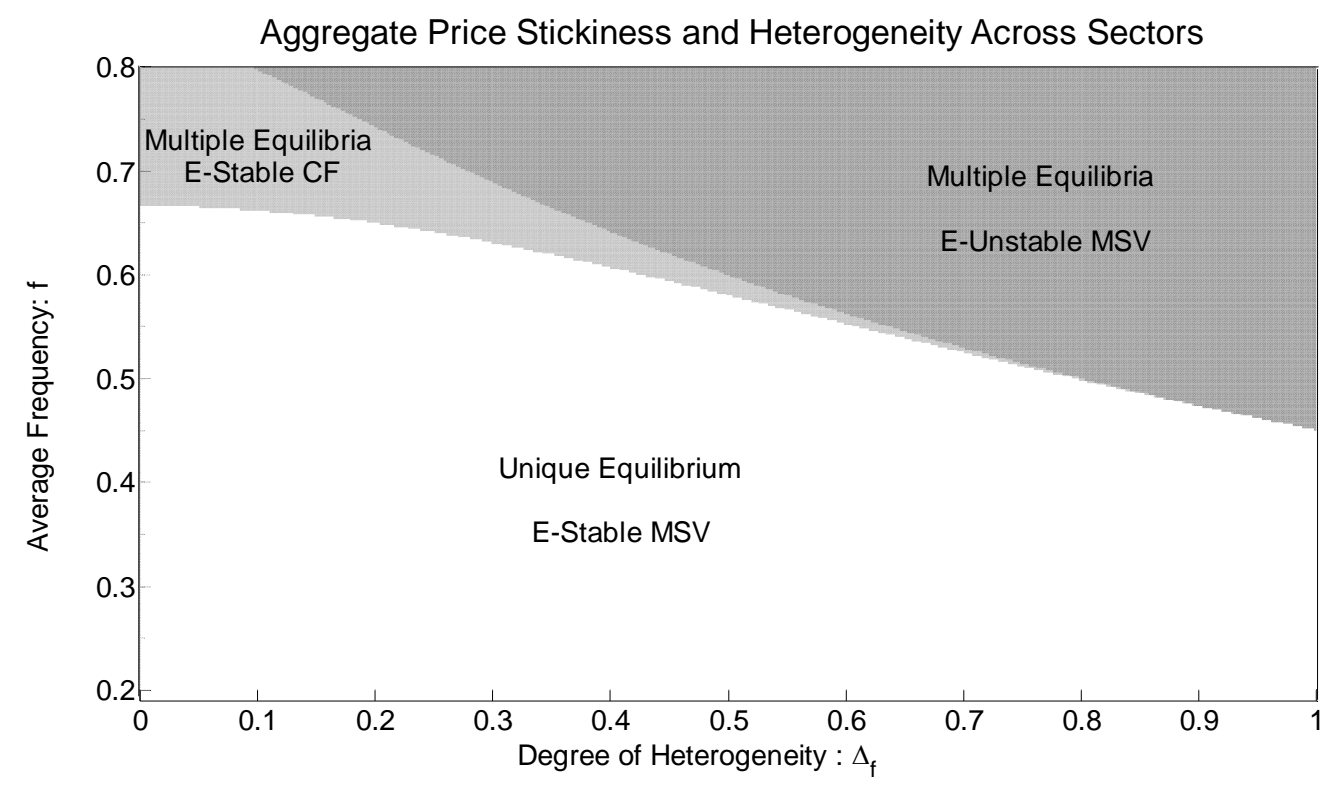

Figure 5: Regions of determinacy and expectational stability when varying $f$ and $\Delta_{f}$. Goods are complements $\xi=0.5$ and the rule is forward-looking with $\phi_{\pi}=2$ and $\omega=\eta=0.5$. All remaining parameters, except for $\gamma^{F}$ and $\gamma^{S}$, are as in Table 1.

Note: $\mathrm{CF}=$ Common Factor representation of a "sunspot" equilibrium and MSV = Minimum State Variable representation of a fundamental equilibrium.

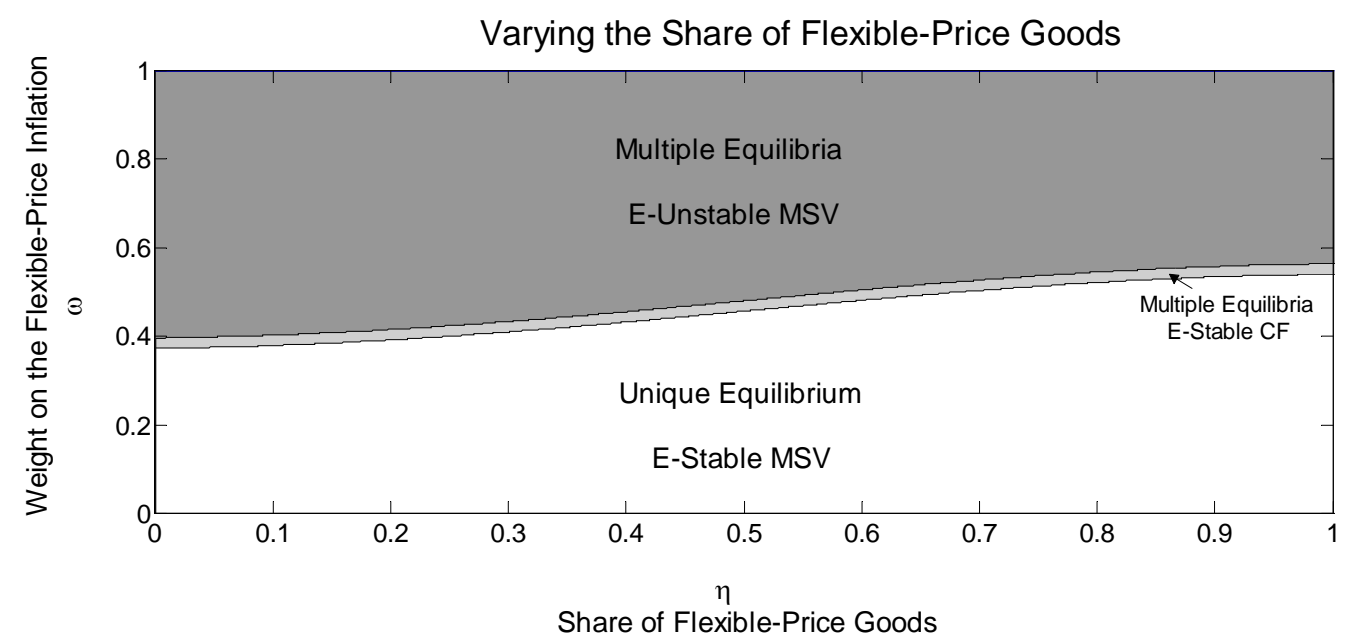

Figure 6: Regions of determinacy and expectational stability when varying $\omega$ and $\eta$. Goods are complements $\xi=0.5$ and the rule is forward-looking with $\phi_{\pi}=2$. All remaining parameters are as in Table 1.

Note: $\mathrm{CF}=$ Common Factor representation of a "sunspot" equilibrium and MSV = Minimum State Variable representation of a fundamental equilibrium. 


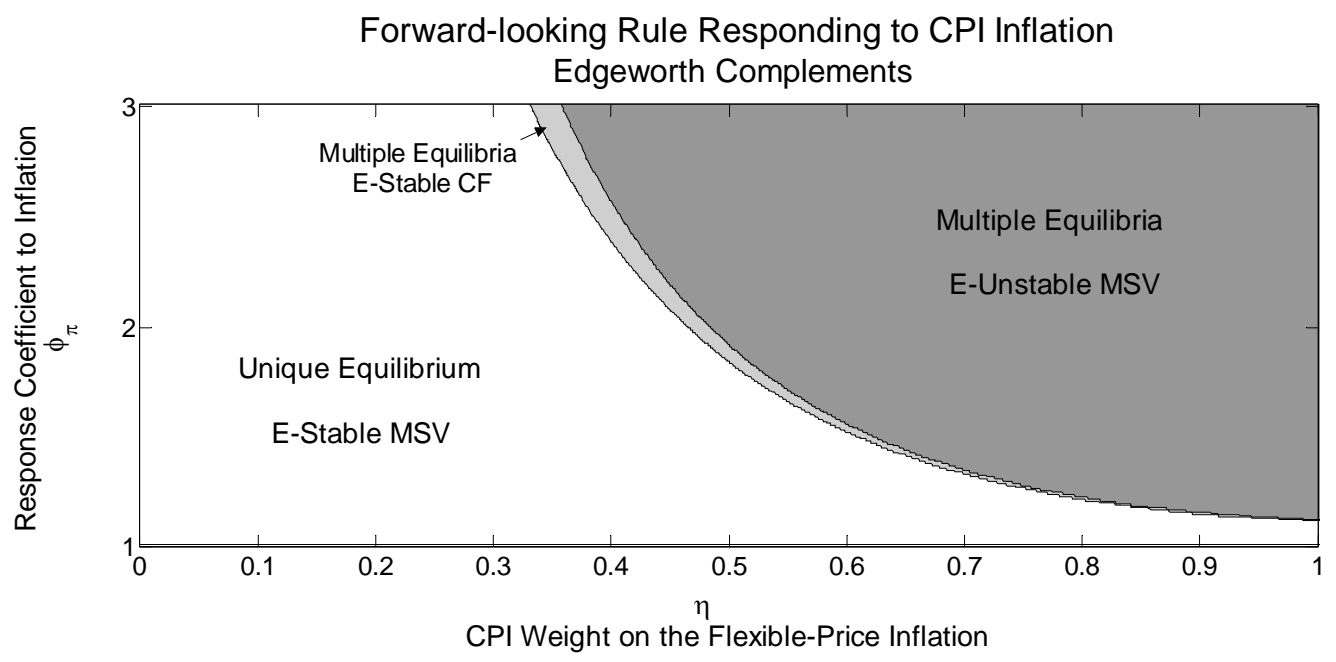

Figure 7: Regions of determinacy and expectational stability for a forward-looking rule that responds to the CPI inflation. Goods are complements $\xi=0.5$. All remaining parameters are as in Table 1. Note: $\mathrm{CF}=$ Common Factor representation of a "sunspot" equilibrium and MSV = Minimum State Variable representation of a fundamental equilibrium.

degree of heterogeneity (i.e., the lower is $\Delta_{f}$ ) above which aggregate instability arises. Third, the equilibrium is always unique and E-stable, independently from the degree of heterogeneity, if the economy is sufficiently sticky in aggregate terms (low $f) .{ }^{27}$

The Share of Flexible-Price Goods and the Response to CPI Inflation Next, we assess how the results depend on the share of flexible price goods in aggregate consumption $\eta$. Note that, in contrast to $\omega$, the share $\eta$ affects the steady state, including the relative price $\bar{q}$. Therefore, it is not longer possible to focus on the steady state where $\bar{q}=1$. As shown in the Appendix A the (log) CPI inflation, which affects the Euler equation (30), is redefined as $\widehat{\pi}_{t}=\eta^{F} \widehat{\pi}_{t}^{F}+\eta^{S} \widehat{\pi}_{t}^{S}$ where $\eta^{F}=\eta\left[\eta+(1-\eta)\left(\frac{1}{\bar{q}}\right)^{1-\xi}\right]^{-1}$ and $\eta^{S}=(1-\eta)\left[\eta \bar{q}^{1-\xi}+(1-\eta)\right]^{-1}$.

Figure 6 presents the results of varying the share $\eta$, assuming the goods are complements and the rule is forward-looking. As $\eta$ decreases the threshold value for $\omega$, beyond which aggregate instability arises, decreases as well. As a result, a rule responding to the expected headline inflation $\widehat{\pi}_{t+1}^{o}=$ $\omega \widehat{\pi}_{t+1}^{F}+(1-\omega) \widehat{\pi}_{t+1}^{S}$ is more prone to induce stability problems, the smaller is the share of flexible

\footnotetext{
${ }^{27}$ This last result holds for a general calibration of the policy rule, and not only for the case considered here, i.e. $\phi_{\pi}=2$ and $\omega=\eta$. Moreover, we find that the determinacy and E-stability areas of Figure 5 shrink (respectively, get larger) if we increase (respectively, decrease) the weight $\omega$. That is, the amounts of price flexibility and of sectoral heterogeneity required to induce aggregate instability are lower when the central bank decides to give more weight to flexible price inflation.
} 
price goods. This figure also shows that in our model responding to the CPI inflation $\widehat{\pi}_{t}$, i.e. $\omega=\eta$, (which corresponds to the 45 degree line) does not guarantee determinacy and/or E-stability.

But what are the effects of responding to CPI inflation while varying the share $\eta$ ? Figure 7 shows the determinacy and E-stability areas with respect to $\phi_{\pi}$ and $\eta$ for complement goods and the policy rule $\widehat{r}_{t}=\phi_{\pi} \mathbb{E}_{t} \widehat{\pi}_{t+1}$. The Figure shows that the results are to some extent qualitatively similar to those obtained for a rule responding to expected headline inflation (see Figure 2). In particular, when responding to CPI inflation, forward-looking rules are more suceptible to non-learnable fundamentaldrive equilibria, the higher the share $\eta$. However, note that there is no threshold for this share beyond which any active rule induces these instability problems, as in the case of responding to the headline inflation.

\section{Robustness and Discussion}

In this Section, we discuss the implications of relaxing some of the assumptions of our set-up, including (i) rules that, besides inflation, also respond to output; (ii) different timings for money in the utility function; and (iii) the approach to learning.

Extended Interest Rate Rules The destabilizing consequences of considering a flexible-price inflation measure persist if we augment those rules with an explicit response to aggregate activity. In particular, for any given coefficient $\phi_{\pi}$, the Appendix D shows that a positive response to (expected) output lowers the upper bound on $\omega$ above which multiple equilibria arise and the fundamental MSVREE ceases to be stable under learning. So aggregate instability is more pervasive. This outcome is in line with the intuition we provided for the analytical results of Propositions 1-3, as well as the numerical results of Subsection C. As shown, a higher $\omega$ implies a more negative implicit response to current sticky-price output, which undermine determinacy and E-stability.

Cash-In-Advance Timing for Money Our model embeds a Cash-When-I'm-Done (CWID) timing where end-of-period money balances, net of current income and consumption, enter the utility function. This is the most frequent timing used in the New-Keynesian literature. But how would our results change if we adopted a Cash in Advance (CIA) timing? that is, if the money that is available to satisfy consumption is the cash left after visiting the bonds market, but before entering the goods market?

In the Appendix D, we show that the determinacy and learnability conditions for a forward-looking rule under the CWID timing are identical to those for a contemporaneous rule under the CIA timing. This is because the CWID Euler equation under a forward-looking rule is identical to the CIA Euler Equation with a contemporaneous rule. This isomorphism was first pointed out by Carlstrom and Fuerst (2001) in a one-sector New-Keynesian model and in the context of equilibrium determinacy. We show that it survives in our two-sector model and, more importantly, that it also applies to the 
learnability conditions.

Therefore, the graphical characterizations of our results in Figure 1 and 2 are also valid for a contemporaneous rule under the CIA timing: that is, active contemporaneous rules responding to inflation measures that include some flexible-price inflation are more prone to induce indeterminacy and non-learnable fundamental equilibria than rules that exclusively respond to sticky-price inflation. ${ }^{28}$

The Euler Equation Approach versus the Infinite Horizon Learning Approach We have adopted the "Euler Equation" learning approach of Evans and Honkapohja (2001), as it remains the most common in the literature on learning and policy rules, including the seminal work of Bullard and Mitra (2002). By selecting this approach, we have assumed that our "boundedly rational" agents make decisions based the a simple marginal costs - marginal benefits analysis captured by the first order conditions obtained under rational expectations. In other words, agents were only required to make one-period-ahead forecasts when making decisions about variables such as consumption, labor and price setting.

The infinite horizon (IH) approach proposed by Preston (2006) is an alternative approach to model learning. Preston suggests that when agents have subjective beliefs about the evolution of aggregate state variables, the decision rules for infinitely-lived optimizing agents should depend on infinite horizon expectations. Hence, in equilibrium, the Euler equation and the New-Keynesian Phillips curve should feature expectations over the infinite future sequences of inflation, real activity and the policy rate. As agents do not know others' beliefs and preferences, they cannot compute the exact law of motion of aggregate variables and therefore long-horizon forecasts are "irreducible" to a one-step-ahead prediction.

We infer, however, that our results might still hold under the IH approach provided some plausible conditions. Our inference is based on Honkapohja et al. (2003). They argue that even under the IH approach, if each agent actually forms the same forecasts - given that everyone is assumed to rely on the same econometric model and to have access to the same historical data - then ex-post all agents behave identically, leading to a symmetric equilibrium. But in a symmetric equilibrium with all agents (correctly) inferring that the market would clear at any point in time, the IH approach collapses to the "Euler equation" approach to learning. For more general conditions, there are no equivalence convergence results between these two approaches. In this regard, it would be interesting to pursue an extensive IH learning analysis of our model, taking into account that in our multiple-sector economy, agents would need to form long-horizon expectations not only of future marginal costs and sectoral inflations, but also of future relative prices. This goes beyond the scope of this paper and we are currently pursuing it as a separate research project.

More generally, once the assumption of rational expectations is dropped, there are other alternative ways to model expectation formation. All that is required is that the latter satisfy standard probability laws at the individual level. For instance, Schorfheide (2005) and Svensson and Williams

\footnotetext{
${ }^{28}$ In principle, given the isomorphism, an active backward-looking rule responding to any measure of inflation should deliver a unique and learnable equilibrium in the CIA model.
} 
(2007) assume Bayesian learning to account for the possibility of, respectively, policy shifts and model uncertainty. More recently, Adam and Marcet (2010) provide a general discussion about the minimal set of requirements for subjective expectations to be consistent with individual optimization.

\section{Conclusions}

We develop a two-sector closed economy model with heterogeneous price stickiness across sectors, to show that the inflation measure included in an interest rate rule is relevant for ensuring equilibrium determinacy and learnability a la Evans and Honkapohja (2001). We find that active rules responding to inflation measures that put some weight on the inflation of the sector with low price stickiness are more suceptible to induce aggregate instability in the economy than rules that respond exclusively to the inflation of the sector with high price stickiness. The sources of instability are related to the presence of equilibria driven by fundamental shocks that are not learnable, equilibria driven by self-validating beliefs that are learnable, and equilibria with unbounded fluctuations.

Our results may provide some guidance for the discussion on the appropriate inflation measure for the monetary policy stance in the context of energy price shocks. Should central banks respond to a headline measure that includes the inflation of flexible energy prices? or, instead, should they react to a core measure that includes mainly the inflation of sticky prices? The results of Bodenstein et al. (2008) advocate for core inflation as they find that, for fundamental-driven equilibria, rules responding to a forecast of headline inflation induce greater volatility in core inflation and the output gap than rules reacting to a forecast of core. Our results for forward-looking rules support this policy recommendation. But our explanation is different: by responding to an inflation measure that includes flexible-price inflation, a rule may induce greater volatility associated with learnable selffulfilling expectations equilibria of the "sunspot" type, and fundamental-driven equilibria that are not learnable by the agents.

While we have focused on price rigidity, there are additional sources of sectoral heterogeneity that one might want to embed into a multi-sector New-Keynesian model. In Airaudo and Zanna (2010), we allow sectors to differ both because of price stickiness and the impact of policy rates on marginal costs of production, i.e., the cost channel of Ravenna and Walsh (2006). ${ }^{29}$ We show that the inflation measure adopted by the central bank is relevant for equilibrium determinacy and Estability both under a forward-looking and a contemporaneous interest rate rule. Another issue is the "durability" of goods. Barsky et al. (2007) show that the aggregate consumption share and the degree of price rigidity of durable goods have strong implications for the monetary policy transmission in New-Keynesian models. Whether durability affects our determinacy and E-stability results is an interesting question to explore.

\footnotetext{
${ }^{29}$ In fact, Llosa and Tuesta (2007) have found that in a typical one-sector New Keynesian model, the cost channel can significantly affect the typical equilibrium determinacy and learnability conditions from Bullard and Mitra (2002).
} 


\section{Appendix}

\section{A. The Steady State}

Consider the general two-sector model. From the definitions of the relative price $\bar{q}=\frac{\bar{P} F}{\bar{P}^{S}}$ and the CPI index (3), we have:

$$
\left(\frac{\bar{P}}{\bar{P}^{S}}\right)^{1-\xi}=\eta \bar{q}^{1-\xi}+1-\eta \text { and }\left(\frac{\bar{P}}{\bar{P}^{F}}\right)^{1-\xi}=\eta+(1-\eta)\left(\frac{1}{\bar{q}}\right)^{1-\xi}
$$

From the relative demands of both goods in (4):

$$
\bar{c}^{S}=(1-\eta)\left[\eta \bar{q}^{1-\xi}+1-\eta\right]^{\frac{\xi}{1-\xi}} \bar{c} \quad \text { and } \quad \bar{c}^{S}=\frac{1-\eta}{\eta} \bar{q}^{\xi} \bar{c}^{F}
$$

Assume that $\varphi^{F}=\varphi^{S} \equiv \varphi \cdot{ }^{30}$ Using equation (10) for $i=F, S, \overline{m c} F=\frac{\mu^{F}-1}{\mu^{F}}, \overline{m c}{ }^{S}=\frac{\mu^{S}-1}{\mu^{S}}$, together with equations (36)-(37), we obtain:

$$
\left(\bar{c}^{F}\right)^{\varphi+\sigma}=\frac{\left(1+v^{F}\right) \eta^{\sigma}\left(\mu^{F}-1\right) \bar{q}^{1-\sigma \xi} \overline{\mathfrak{g}}}{A \mu^{F}} \quad \text { and } \quad\left(\bar{c}^{S}\right)^{\varphi+\sigma}=\frac{\left(1+v^{S}\right)(1-\eta)^{\sigma}\left(\mu^{S}-1\right) \overline{\mathfrak{g}}}{A \mu^{S}},
$$

with $\overline{\mathfrak{g}}=\left[\eta \bar{q}^{1-\xi}+1-\eta\right]^{\frac{1-\xi \sigma}{\xi-1}}$. Combining these equations with $\frac{\bar{c}^{S}}{\bar{c}^{F}}=\frac{1-\eta}{\eta} \bar{q}^{\xi}$, we obtain:

$$
\bar{q}^{1+\xi \varphi}=\frac{\left(1+v^{S}\right)}{\left(1+v^{F}\right)}\left(\frac{\mu^{S}-1}{\mu^{F}-1}\right)\left(\frac{\mu^{F}}{\mu^{S}}\right)\left(\frac{\eta}{1-\eta}\right)^{\varphi} .
$$

Clearly the steady-state relative price is $\bar{q}=1$, if and only if $\frac{\left(1+v^{S}\right)}{\left(1+v^{F}\right)}\left(\frac{\mu^{S}-1}{\mu^{F}-1}\right)\left(\frac{\mu^{F}}{\mu^{S}}\right)\left(\frac{\eta}{1-\eta}\right)^{\varphi}=1$. Given calibrated values for $\mu^{F}, \mu^{S}, \eta$, and $\varphi$, we can pick $v^{S}$ and $v^{F}$ such that $\bar{q}=1$.

For the strongly dichotomous economy, the procedure is unchanged. With $\mu^{F} \rightarrow+\infty$, equation (39) reduces to $\bar{q}^{1+\xi \varphi}=\frac{\left(1+v^{S}\right)}{\left(1+v^{F}\right)}\left(\frac{\mu^{S}-1}{\mu^{S}}\right)\left(\frac{\eta}{1-\eta}\right)^{\varphi}$.

Note that the steady-state value of $\bar{q}$ affects the log-linearization of the CPI inflation. From (3), we can obtain the following expression for the (log) CPI inflation $\widehat{\pi}_{t}=\eta^{F} \widehat{\pi}_{t}^{F}+\eta^{S} \widehat{\pi}_{t}^{S}$, where $\eta^{F}=\eta\left[\eta+(1-\eta)\left(\frac{1}{\bar{q}}\right)^{1-\xi}\right]^{-1}$ and $\eta^{S}=(1-\eta)\left[\eta \bar{q}^{1-\xi}+(1-\eta)\right]^{-1}$. This expression reduces to $\widehat{\pi}_{t}=\eta \widehat{\pi}_{t}^{F}+(1-\eta) \widehat{\pi}_{t}^{S}$ if we let $\bar{q}=1$.

\section{B. Lemmata and Proofs of the Propositions}

\section{B.1. Lemmata}

Lemma 1 Let $\tau \equiv \alpha \theta$. a) If $\xi \leq \frac{1}{\sigma}$ (Edgeworth complements or neutral) then $\tau \geqq 1$; b) if $\xi>\frac{1}{\sigma}$ (Edgeworth substitutes) then $0<\tau<1$.

\footnotetext{
${ }^{30}$ This assumption greatly simplifies the algebra and the notation.
} 
Proof. The proof is available from the authors upon request.

Lemma 2 Consider the model $\widehat{x}_{t}=\Omega \mathbb{E}_{t} \widehat{x}_{t+1}+\Pi \widehat{z}_{t}$, where $\widehat{x}_{t}$ is a $2 \times 1$ vector of non-predetermined endogenous variables, $\Omega$ and $\Pi$ are conformable matrices, and $\widehat{z}_{t}$ is an exogenous shock following $\widehat{z}_{t}=\psi \widehat{z}_{t-1}+\nu_{t}^{S}$, with $\psi \in(0,1)$, and $\nu_{t}^{S} \backsim \operatorname{Normal}\left(0, \varrho^{2}\right)$. Define the determinant of $\Omega-I$ as $\operatorname{Det}(\Omega-I)$ where $I$ is the identity matrix.

a) if $\operatorname{Det}(\Omega-I)>0$ and Trace $(\Omega-I)<0$, then the $M S V$ representation of an equilibrium $\widehat{x}_{t}=\mathcal{A}+\mathcal{N} \widehat{z}_{t}$ with $\left(0_{2 \times 1}, \overline{\mathcal{N}}\right)$ is E-stable.

b) if $\operatorname{Det}(\Omega-I)<0$, then the $M S V$ representation of an equilibrium $\widehat{x}_{t}=\mathcal{A}+\mathcal{N} \widehat{z}_{t}$ with $\left(0_{2 \times 1}, \overline{\mathcal{N}}\right)$ is E-unstable.

c) Provided that the model displays indeterminacy of order one, if the eigenvalues of $\Omega$ are real, $\operatorname{Det}(\Omega-I)>0$, and Trace $(\Omega-I)<0$, then the CF representation of a "sunspot" $\widehat{x}_{t}=\mathcal{A}+\mathcal{N} \widehat{z}_{t}+\mathcal{G} \varepsilon_{t}$, with $\left(0_{2 \times 1}, \overline{\mathcal{N}}, \overline{\mathcal{G}}\right)$ is E-stable, where $\varepsilon_{t}$ is the "sunspot" following $\varepsilon_{t}=v \varepsilon_{t-1}+\varpi_{t}$ with $|v|<1$, and $\varpi_{t}$ is an arbitrary martingale difference sequence.

Proof. To prove a), recall (17). Since the model has no lagged terms and $\psi \in(0,1)$, then the MSV solution is E-stable, if all the eigenvalues $e_{1}$ and $e_{2}$ of $D T_{\mathcal{A}}=\Omega$ have real parts less than one or, equivalently, if all the eigenvalues $u_{1}$ and $u_{2}$ of $\Omega-I$ have negative real parts. Sufficient conditions for this are $\operatorname{Det}(\Omega-I)>0$ and $\operatorname{Trace}(\Omega-I)<0$, given that the eigenvalues must satisfy $\operatorname{Det}(\Omega-I)=u_{1} u_{2}$ and $\operatorname{Trace}(\Omega-I)=u_{1}+u_{2}$.

For b), it suffices to prove that the E-stability conditions are violated. But this is the case if $\operatorname{Det}(\Omega-I)<0$, since this implies that there exists one eigenvalue with a positive real part.

Finally to prove c), recall (18). Given that by assumption the model displays indeterminacy of order one, that is $\Omega$ has only one non-explosive eigenvalue, then pick " $v$ " to be that eigenvalue; so $v=e$ with $|e|<1$. Since $\psi \in(0,1)$ and $v=e$ with $|e|<1$, then the CF representation is E-stable if all the eigenvalues $e_{1}$ and $e_{2}$ of $D T_{\mathcal{A}}=\Omega$ have real parts less than one, or equivalently if all the eigenvalues $u_{1}$ and $u_{2}$ of $\Omega-I$ have negative real parts. As in a), this is satisfied if $\operatorname{Det}(\Omega-I)>0$ and $\operatorname{Trace}(\Omega-I)<0$.

Lemma 3 Let $\omega^{*} \equiv \frac{1}{\tau}, \omega^{u} \equiv \omega^{*}\left(1-\frac{\alpha}{2} \phi_{y}\right), \omega^{\prime} \equiv \omega^{*}\left[1-\frac{\alpha \beta \phi_{y}}{(1+\beta)}\right], \phi_{2}(\omega) \equiv \frac{1}{\tau \omega}, \phi_{3}(\omega) \equiv \frac{\delta \alpha+2(1+\beta)}{\delta \alpha+2(1+\beta) \tau \omega}-$ $\frac{\alpha(1+\beta)}{\delta \alpha+2(1+\beta) \tau \omega} \phi_{y}$, and $\phi_{4}(\omega) \equiv\left(1-\frac{\alpha \beta \phi_{y}}{(1+\beta)}\right) \phi_{2}(\omega)$. Assume $\omega \in(0,1)$. If $0<\phi_{y}<\frac{2}{\alpha}$, then $\omega^{u}<\omega^{\prime}<\omega^{*}$ and the following properties for $\phi_{2}(\omega), \phi_{3}(\omega)$, and $\phi_{4}(\omega)$ hold:

a) $\phi_{2}(\omega): \lim _{\omega \rightarrow 0} \phi_{2}(\omega)=+\infty, \lim _{\omega \rightarrow 1} \phi_{2}(\omega)=\frac{1}{\tau}<1, \frac{\partial \phi_{2}(\omega)}{\partial \omega}<0$ and $\phi_{2}(\omega) \gtreqless 1$ if and only if $\omega \lesseqgtr \omega^{*}$.

b) $\phi_{3}(\omega): \lim _{\omega \rightarrow 0} \phi_{3}(\omega)=1+\frac{2(1+\beta)}{\delta \alpha}-\frac{(1+\beta)}{\delta} \phi_{y}>1, \lim _{\omega \rightarrow 1} \phi_{3}(\omega)<1, \frac{\partial \phi_{3}(\omega)}{\partial \omega}<0$ and $\phi_{3}(\omega) \gtreqless 1$ if and only if $\omega \lesseqgtr \omega^{u}$ where $\omega^{u} \in\left(0, \omega^{*}\right)$. 
c) $\phi_{4}(\omega): 0<\phi_{4}(\omega)<\phi_{2}(\omega)$ for any $\omega \in(0,1), \frac{\partial \phi_{4}(\omega)}{\partial \omega}<0, \phi_{4}(\omega) \gtreqless 1$ if and only if $\omega \lesseqgtr \omega^{\prime}$, where $\omega^{\prime} \in\left(\omega^{u}, \omega^{*}\right)$, and $\phi_{3}(\omega)<\phi_{4}(\omega)$ for any $\omega \in\left(0, \omega^{\prime}\right)$.

Proof. The proof is available from the authors upon request.

\section{B.2. Proof of Proposition 1}

Proof. Using equations (6), (22), (25), (28), and the rule (19)-(20), the equilibrium dynamics around the non-stochastic steady state are described by the following system:

$$
\widehat{x}_{t}=\Upsilon+\Omega \mathbb{E}_{t} \widehat{x}_{t+1}+\Pi \widehat{z}_{t} \quad \text { and } \quad \widehat{z}_{t}=\psi \widehat{z}_{t-1}+\nu_{t}
$$

where $\widehat{x}_{t}=\left[\widehat{\pi}_{t}^{S}, \widehat{c}_{t}^{S}\right]^{\prime}, \Upsilon=[0,0]^{\prime}$,

$$
\Omega=\left[\begin{array}{cc}
\beta+\frac{\alpha \delta\left(1-\phi_{\pi}\right)}{\left(1-\tau \omega \phi_{\pi}\right)} & \delta \\
\frac{\alpha\left(1-\phi_{\pi}\right)}{\left(1-\tau \omega \phi_{\pi}\right)} & 1
\end{array}\right],
$$

and the form of $\Pi$ is omitted since it is not required for the analysis. As there are no lagged endogenous variables $\left(\widehat{x}_{t-1}\right)$ in the system (40), we can use it for both the equilibrium determinacy and learning analyses, noting that the matrix $J$ from the representation in (15) satisfies $J=\Omega^{-1}$.

To assess equilibrium determinacy it is sufficient to characterize the eigenvalues of $\Omega$ defined in $(41) .{ }^{31}$ Let $\phi_{2}(\omega) \equiv \frac{1}{\tau \omega}$. The characteristic polynomial associated with $\Omega$ is given by $\mathcal{P}(e)=e^{2}-$ $\operatorname{Trace}(\Omega) e+\operatorname{Det}(\Omega)$ with

$$
\begin{gathered}
\operatorname{Det}(\Omega)=\beta \\
\mathcal{P}(1)=\frac{\alpha \delta\left(\phi_{\pi}-1\right)}{\tau \omega\left[\phi_{2}(\omega)-\phi_{\pi}\right]},
\end{gathered}
$$

and

$$
\mathcal{P}(-1)=\frac{[2(1+\beta) \tau \omega+\alpha \delta]}{\tau \omega}\left[\frac{\phi_{1}(\omega)-\phi_{\pi}}{\phi_{2}(\omega)-\phi_{\pi}}\right] .
$$

To prove a) use Lemma 1 and $\beta, \alpha, \delta>0$ to deduce that

$$
1<\phi_{1}(\omega)<\phi_{2}(\omega)
$$

for any $\omega \in(0,1)$ when $\frac{1}{\sigma} \leq \xi$, or any $\omega \in\left(0, \omega^{*}\right)$ when $\frac{1}{\sigma}>\xi$. Next use (42)-(45) to derive that if $1<\phi_{\pi}<\phi_{1}(\omega)$ then $\mathcal{P}(1)>0, \mathcal{P}(-1)>0$, and $\operatorname{Det}(\Omega) \in(0,1)$. By Azariadis (1993), these are sufficient conditions for the roots of $\mathcal{P}(e)=0$ to be inside of the unit circle. Since there are two non-predetermined variables, $\widehat{\pi}_{t}^{S}$ and $\widehat{c}_{t}^{S}$, then by Blanchard and Kahn (1980) we conclude that there exists a unique equilibrium.

On the other hand, if $\phi_{\pi}>\phi_{1}(\omega)$ then we need to analyze two cases. In the first one: $\phi_{1}(\omega)<$ $\phi_{\pi}<\phi_{2}(\omega)$, which together with (43)-(45) imply that $\mathcal{P}(1)>0$ and $\mathcal{P}(-1)<0$. In the second case:

\footnotetext{
${ }^{31}$ To apply the results of Blanchard and Kahn (1980) for the determinacy analysis, we argued in the main text that the model needs to be written as in (15). However, recall that $J=\Omega^{-1}$, provided that the inverse exists. Hence the eigenvalues of $J$ are the reciprocals of the eigenvalues of $\Omega$.
} 
$\phi_{2}(\omega)<\phi_{\pi}$, which in tandem with (43)-(45) imply that $\mathcal{P}(1)<0$ and $\mathcal{P}(-1)>0$. Hence, following Azariadis (1993), we can infer that under both cases, there is one root of $\mathcal{P}(e)=0$ inside the unit circle, while the other one is outside of it. Since there are two non-predetermined variables, we conclude that there exist multiple equilibria. Note that in both cases the roots of $\mathcal{P}(e)=0$ that correspond to the eigenvalues of $\Omega$ are real since $\mathcal{P}(-1)<0$ or $\mathcal{P}(1)<0 .{ }^{32}$

To prove b) observe that when $\frac{1}{\sigma}>\xi$, we have that $\tau>1$, by Lemma 1 . Hence, for any $\omega \in\left(\omega^{*}, 1\right)$, with $\omega^{*}=\frac{1}{\tau}, \phi_{2}(\omega)<\phi_{1}(\omega)<1$. Then for any $\omega \in\left(\omega^{*}, 1\right)$, assuming $\phi_{\pi}>1$ implies that $\phi_{\pi}>\phi_{1}(\omega)$ and $\phi_{\pi}>\phi_{2}(\omega)$. Use these and (43)-(45) to derive that $\mathcal{P}(1)<0$ and $\mathcal{P}(-1)>0$. These imply that one root of $\mathcal{P}(e)=0$ is inside the unit circle, whereas the other one is outside of it. As there are two non-predetermined variables, we conclude that there exist multiple equilibria. Note that once more the roots of $\mathcal{P}(e)=0$ are real, since $\mathcal{P}(1)<0$.

\section{B.3. Proof of Proposition 2}

Proof. First use the definition of $\Omega$ in (41) to derive

$$
\begin{gathered}
\operatorname{Trace}(\Omega-I)=\beta-1+\frac{\alpha \delta\left(1-\phi_{\pi}\right)}{\tau \omega\left[\phi_{2}(\omega)-\phi_{\pi}\right]} \text { and } \\
\operatorname{Det}(\Omega-I)=-\frac{\alpha \delta\left(1-\phi_{\pi}\right)}{\tau \omega\left[\phi_{2}(\omega)-\phi_{\pi}\right]},
\end{gathered}
$$

where $I$ is the identity matrix. Next, note that by Lemma 1 and $\beta, \alpha, \delta>0$, we know that $1<\phi_{1}(\omega)<$ $\phi_{2}(\omega)$, for any $\omega \in(0,1)$ when $\frac{1}{\sigma} \leq \xi$, or any $\omega \in\left(0, \omega^{*}\right)$ when $\frac{1}{\sigma}>\xi$. Hence, if $\phi_{1}(\omega)<\phi_{\pi}<\phi_{2}(\omega)$ then by part a2) of Proposition 1 we know that there is indeterminacy (multiple equilibria), which is of order one. Moreover, as discussed in the proof of Proposition 1, the eigenvalues of $\Omega$ are real. Furthermore, by (46) and (47), when $\phi_{1}(\omega)<\phi_{\pi}<\phi_{2}(\omega)$ and $\phi_{\pi}>1$, we have that $\operatorname{Trace}(\Omega-I)<0$ and $\operatorname{Det}(\Omega-I)>0$. Using these we can apply part c) of Lemma 2, to conclude that the CF representation of stationary "sunspots" is E-stable.

\section{B.4. Proof of Proposition 3}

Proof. First recall (46) and (47) from the proof of Proposition 2.

To prove a) use (46) and (47) to deduce that if $1<\phi_{\pi}<\phi_{2}(\omega)$, then Trace $(\Omega-I)<0$ and $\operatorname{Det}(\Omega-I)>0$. Then apply part a) of Lemma 2 to conclude that the MSV solution is E-stable. On the other hand, if $\phi_{\pi}>1$ and $\phi_{\pi}>\phi_{2}(\omega)$, we have that $\operatorname{Det}(\Omega-I)<0$, by $(47)$. Then use this and part b) of Lemma 2 to conclude that the MSV solution is E-unstable.

For part b) note that when $\frac{1}{\sigma}>\xi$, Lemma 1 implies that $\tau>1$. Therefore for any $\omega \in\left(\omega^{*}, 1\right)$, with $\omega^{*}=\frac{1}{\tau}, \phi_{2}(\omega)<1$. Hence $\phi_{\pi}>\phi_{2}(\omega)$, since $\phi_{\pi}>1$. This together with $(47)$ mean that $\operatorname{Det}(\Omega-I)<0$. And by part b) of Lemma 2, we conclude that the MSV solution is E-unstable.

\footnotetext{
${ }^{32}$ See Azariadis (1993).
} 


\section{Calibration of Price Stickiness}

To calibrate the price adjustment cost coefficients $\gamma^{F}$ and $\gamma^{S}$, we use the micro-evidence for the U.S. by Bils and Klenow (2004). There are, however, a few caveats:

1. Aggregation bias: Bils and Klenow refer to a sample of 350 categories of goods and services, while our model is a two-sector two-good economy. Therefore, going from the micro-evidence to the macro model may induce some aggregation bias. Extending the model to incorporate 350 sectors would indeed help address this bias, but will make the model intractable, without adding further insights to our discussion on the inflation measure. Hence, we keep the two-sector set-up and calibrate $\gamma^{F}$ and $\gamma^{S}$ to match some key moments of the Bils and Klenow's evidence. ${ }^{33}$

2. Frequency: Bils and Klenow's statistics are at monthly frequencies, while a quarterly calibration is preferable to compare our results to those of the standard one-sector New-Keynesian literature. To obtain a quarterly calibration we elaborate on their Table A1, which reports the monthly frequency of price changes. We compute the per-quarter average frequency and expected duration of price changes. As in Carvalho (2006), for each sector $i=1,2, \ldots, 350$, we set the per month probability of a price change, $\theta^{i}$, equal to the observed frequency (third column of the table). ${ }^{34}$ The per quarter probability of a price change for sector $i$ is given by $f^{i} \equiv \theta^{i}\left[\left(\theta^{i}\right)^{2}+3\left(1-\theta^{i}\right)\right]$. This is obtained by summing up the probability of observing a price change with respect to the previous quarter in at least one of the months of the current quarter. Then the expected duration between price spells in sector $i$ is simply $d^{i}=\left(f^{i}\right)^{-1}$ quarters. Doing this for each sector, we obtain the (weighted) average quarterly frequency of price change, $f$, and the (weighted) average quarterly duration of price rigidity, $d$ :

$$
f \equiv \sum_{i=1}^{350} w_{i} f^{i} \text { and } d \equiv \sum_{i=1}^{350} w_{i} d^{i},
$$

where $w_{i}$ is the relative weight of sector $i$ in the Bils and Klenow's sample. The weights are computed by using the cumulative density in the last column of their table. We obtain that $f=0.52$ and $d=2.7$; that is, on average, firms change their price at least once with $52 \%$ probability per quarter, while the expected duration is between 2 and 3 quarters, after a price change. ${ }^{35}$

\footnotetext{
${ }^{33}$ Carvalho (2006) extensively discusses the quantitative consequences of the aggregation bias. Nevertheless, for our analysis, aggregation has a great advantage. In a two-sector model, the measure of inflation targeted by the policy-maker is completely identified by the single weight $\omega \in[0,1]$. On the other hand, in a more general $N$-sector model, this would require considering all possible combinations of sectoral weights $\omega_{1}, \omega_{2}, \ldots, 1-\sum_{i=1}^{N-1} \omega_{i}$, with $\omega_{i} \in[0,1]$ for all $i^{\prime}$ s.

${ }^{34}$ This is based on the assumption that the probability of price changes is independent across all firms in the economy.

${ }^{35}$ An alternative procedure is proposed by Carvalho and Dam (2010). They aggregate the Bils and Klenow's categories into eight sectors, with sector $i$ grouping goods and services with an average duration between price spells of at most $i$ quarters, for $i=1,2, . .7$. The eighth sector includes all remaining categories with a duration above seven quarters. Each sector is then assigned a duration $d^{i}=i$, and a weight $w_{i}$ corresponding to the sum of the relative weights of its components. Simple calculation gives $d \equiv \sum_{i=1}^{8} w_{i} d^{i}=2.55$. The value of $f$ can then by obtained by setting $f^{i}=\left(d^{i}\right)^{-1}$ :
} 
3. Mapping frequencies into price adjustment costs: Assigning values to the adjustment cost coefficients requires two additional steps. First, we compute the quarterly frequencies of price changes in sector $F$ and sector $S$-denoted, respectively, by $f^{F}$ and $f^{S}$ - to match $f=0.52$ and $d=2.7$; that is, $\eta f^{F}+(1-\eta) f^{S}=0.52$ and $\eta d^{F}+(1-\eta) d^{S}=2.7$, where $d^{i} \equiv\left(f^{i}\right)^{-1}$ is sector $i$ 's expected price duration and $\eta$ is the size of the relatively more flexible sector $F$. In pursuing this, we set $\eta=0.4$, which corresponds to the share of firms in the sample for which the expected price duration is equal or smaller than one quarter. Second, from (12), we derive sector $i$ 's reduced-form augmented Phillips Curve with respect to its marginal cost:

$$
\widehat{\pi}_{t}^{i}=\beta \mathbb{E}_{t} \widehat{\pi}_{t+1}^{i}+\kappa^{i} \widehat{m c}_{t}^{i}, \text { for } i=F, S,
$$

where $\kappa^{i} \equiv \frac{\mu^{i}-1}{\gamma^{i}(\bar{\pi})^{2}}, m c_{t}^{i} \equiv \frac{M C_{t}^{i}}{P_{t}^{i}}$ and $\widehat{m c} c_{t}^{i}$ its log-deviation. Under Calvo pricing, we would obtain an identical curve, but with $\kappa^{i} \equiv \frac{\left(1-\mathfrak{p}^{i}\right)\left(1-\beta \mathfrak{p}^{i}\right)}{\mathfrak{p}^{i}}$, where $\mathfrak{p}^{i}=1-f^{i}$ corresponds to the probability of no price change. We exploit this reduced-form equivalence between the Rotemberg's and the Calvo's approach: given the computed value $f^{i}$, we set the adjustment cost coefficient $\gamma^{i}$ such that $\frac{\left(\mu^{i}-1\right)}{\gamma^{i}\left(\bar{\pi}^{i}\right)^{2}}=\frac{\left(1-\mathfrak{p}^{i}\right)\left(1-\beta \mathfrak{p}^{i}\right)}{\mathfrak{p}^{i}}$. This procedure gives us $\gamma^{F}=0.67$ and $\gamma^{S}=74$.

\section{Robustness}

In this Appendix, we explore the robustness of our results to i) the addition of an explicit response to output in the (forward-looking) interest rate rule and ii) a different timing of money in the utility function.

\section{D.1. Responding to Output}

Consider first the strong dichotomy case of Subsection B. Adding an explicit response to expected sticky-price output may aggravate the endogenous instability problems induced by a rule. The following proposition proves the result for the case of Edgeworth complementarity. ${ }^{36}$

Proposition 4 Assume goods are complements and the following forward-looking rule:

$$
\widehat{r}_{t}=\phi_{\pi} \mathbb{E}_{t}\left[\omega \widehat{\pi}_{t+1}^{F}+(1-\omega) \widehat{\pi}_{t+1}^{S}\right]+\phi_{y} \mathbb{E}_{t} \widehat{y}_{t+1}^{S},
$$

\footnotetext{
$f \equiv \sum_{i=1}^{8} w_{i} f^{i}=0.6$. This approach gives then a lower average expected duration and a higher average frequency of price change, i.e. an economy with a lower degree of aggregate price stickiness. A calibration of the adjustment cost coefficients $\gamma^{F}$ and $\gamma^{S}$ based on this moments would actually reinforce our results on the importance of the measure of inflation for determinacy and E-stability.

${ }^{36}$ The restriction to the case of complementarity is without loss of generality. Considering only the sticky-price output in the rule simplifies the analysis. However, as shown in equation (21), the two consumption goods, and therefore sectoral outputs, are proportionally related. Consequently, our results would not qualitatively change, if we considered rules responding to some aggregate measure of output. Details are available from the authors upon request.
} 
with $\phi_{\pi}>1, \omega \in(0,1)$, and $\phi_{y}>0$. Let $\omega^{*} \equiv \frac{1}{\tau}, \phi_{2}(\omega) \equiv \frac{1}{\tau \omega}, \phi_{3}(\omega) \equiv \frac{\delta \alpha+2(1+\beta)}{\delta \alpha+2(1+\beta) \tau \omega}-\frac{\alpha(1+\beta)}{\delta \alpha+2(1+\beta) \tau \omega} \phi_{y}$, and $\omega^{u} \equiv \omega^{*}\left[1-\frac{\alpha \phi_{y}}{2}\right]$.

a) If $0<\phi_{y}<\frac{2}{\alpha}$, then there exists a unique local rational expectations equilibrium and its $M S V$ representation is learnable (E-stable), when $1<\phi_{\pi}<\phi_{3}(\omega)$ for $\omega \in\left(0, \omega^{u}\right)$, where $\omega^{u}<\omega^{*}$.

b) If $\phi_{y} \geq \frac{2}{\alpha}$ then 1) there exist multiple local rational expectations equilibria for any $\phi_{\pi}>1$; 2) the $C F$ representation of stationary "sunspot" equilibria is learnable (E-stable) if $1<\phi_{\pi}<\phi_{2}(\omega)$ for any $\omega \in(0,1)$, and 3) the MSV-REE is non-learnable (E-unstable) when $\phi_{\pi}>\phi_{2}(\omega)$ for any $\omega \in(0,1)$.

Furthermore, $\frac{\partial \omega^{u}}{\partial \phi_{y}}<0$ and $\frac{\partial \phi_{3}(\omega)}{\partial \phi_{y}}<0$.

Proof. Combine the rule (48) with (6), (22), (25), (28) and market clearing $\widehat{y}_{t}^{S}=\widehat{c}_{t}^{S}$ to obtain the system (40) where $\widehat{x}_{t}=\left[\widehat{\pi}_{t}^{S}, \widehat{c}_{t}^{S}\right]^{\prime}, \Upsilon=[0,0]^{\prime}$ and

$$
\Omega=\left[\begin{array}{cc}
\beta+\frac{\alpha \delta\left(1-\phi_{\pi}\right)}{\left(1-\tau \omega \phi_{\pi}\right)} & \delta\left(1-\frac{\alpha \phi_{y}}{\left(1-\tau \omega \phi_{\pi}\right)}\right) \\
\frac{\alpha\left(1-\phi_{\pi}\right)}{\left(1-\tau \omega \phi_{\pi}\right)} & 1-\frac{\alpha \phi_{y}}{\left(1-\tau \omega \phi_{\pi}\right)}
\end{array}\right]
$$

Observe that $\Omega$ collapses to (41), if $\phi_{y}=0$. The characteristic polynomial for $\Omega$ is given by $\mathcal{P}(e)=$ $e^{2}-\operatorname{Trace}(\Omega) e+\operatorname{Det}(\Omega)$ with

$$
\mathcal{P}(1)=\frac{\alpha\left[\delta\left(\phi_{\pi}-1\right)+\phi_{y}(1-\beta)\right]}{\omega \tau\left[\phi_{2}(\omega)-\phi_{\pi}\right]}, \quad \mathcal{P}(-1)=\left[\frac{\delta \alpha+2(1+\beta) \omega}{\omega \tau}\right]\left[\frac{\phi_{3}(\omega)-\phi_{\pi}}{\phi_{2}(\omega)-\phi_{\pi}}\right]
$$

and

$$
\operatorname{Det}(\Omega)=\beta\left\{1-\frac{\alpha \phi_{y}}{\omega \tau\left[\phi_{2}(\omega)-\phi_{\pi}\right]}\right\},
$$

where $\phi_{2}(\omega) \equiv \frac{1}{\omega \tau}$ and $\phi_{3}(\omega) \equiv \frac{\delta \alpha+2(1+\beta)}{\delta \alpha+2(1+\beta) \tau \omega}-\frac{\alpha(1+\beta)}{\delta \alpha+2(1+\beta) \tau \omega} \phi_{y}$. Given the assumption of Edgeworth complementarity $\left(\frac{1}{\sigma}>\xi\right)$, we have that $\tau>1$ by Lemma 1 .

To prove a), first define $\phi_{4}(\omega) \equiv\left(1-\frac{\alpha \beta \phi_{y}}{(1+\beta)}\right) \phi_{2}(\omega)$. By this definition, we have that $\phi_{2}(\omega)<\phi_{4}(\omega)$ if $0<\phi_{y}<\frac{2}{\alpha}$. In addition, by Lemma 3 we know that if $0<\phi_{y}<\frac{2}{\alpha}$ then $\omega^{u}<\omega^{\prime}<\omega^{*}$. Using this and the properties of $\phi_{2}(\omega), \phi_{3}(\omega)$, and $\phi_{4}(\omega)$ from this Lemma, we can also infer that if $\omega \in\left(0, \omega^{u}\right)$ then $\phi_{2}(\omega)>1, \phi_{3}(\omega)>1, \phi_{4}(\omega)>1$, and $\phi_{3}(\omega)<\phi_{4}(\omega)$. In turn, this last inequality, $\phi_{2}(\omega)<\phi_{4}(\omega)$, and the assumption $1<\phi_{\pi}<\phi_{3}(\omega)$ imply the following ranking for $\phi_{\pi}$ and the bounds: $1<\phi_{\pi}<\phi_{3}(\omega)<$ $\phi_{4}(\omega)<\phi_{2}(\omega)$ provided that $\omega \in\left(0, \omega^{u}\right)$. Applying these inequalities to (50) we obtain $\mathcal{P}(1)>0$ and $\mathcal{P}(-1)>0$. Moreover, it is possible to use (51) and show that as long as $1<\phi_{\pi}<\phi_{4}(\omega)$ we have that $\operatorname{Det}(\Omega) \in(0,1)$. Hence for any $\omega \in\left(0, \omega^{u}\right)$, if $0<\phi_{y}<\frac{2}{\alpha}$ and $1<\phi_{\pi}<\phi_{3}(\omega)$ then $\mathcal{P}(1)>0$, $\mathcal{P}(-1)>0$, and $\operatorname{Det}(\Omega) \in(0,1)$. By Azariadis (1993), these are sufficient conditions for the roots of $\mathcal{P}(e)=0$ to be inside of the unit circle. Since there are two non-predetermined variables, $\widehat{\pi}_{t}^{S}$ and $\widehat{c}_{t}^{S}$, then by Blanchard and Kahn (1980) we conclude that there exists a unique equilibrium. 
To prove that under a unique equilibrium its MSV representation is E-stable, we use part a) of Lemma 2. From (49) we can derive that

$$
\operatorname{Trace}(\Omega-I)=\beta-1+\frac{\alpha \delta\left(1-\phi_{\pi}\right)-\alpha \phi_{y}}{\tau \omega\left[\phi_{2}(\omega)-\phi_{\pi}\right]} \quad \text { and } \operatorname{Det}(\Omega-I)=\frac{\alpha\left[\delta\left(\phi_{\pi}-1\right)+\phi_{y}(1-\beta)\right]}{\tau \omega\left[\phi_{2}(\omega)-\phi_{\pi}\right]} .
$$

But as argued before, when $\omega \in\left(0, \omega^{u}\right)$ we have that $\phi_{3}(\omega)<\phi_{2}(\omega)$. Combining this inequality and the assumption $1<\phi_{\pi}<\phi_{3}(\omega)$ together with (52) leads to $\operatorname{Trace}(\Omega-I)<0$ and $\operatorname{Det}(\Omega-I)>0$. Then apply part a) of Lemma 2 to conclude that the MSV representation is E-stable.

To prove b1) consider the following two cases. First, when $\phi_{y} \geq \frac{2}{\alpha}$ then by the definition of $\phi_{3}(\omega)$ we can infer that $\phi_{3}(\omega)<1$. And since by assumption $\phi_{\pi}>1$, we have that if $\phi_{\pi}<\phi_{2}(\omega)$ then the expressions in (50) imply that $\mathcal{P}(1)>0$ and $\mathcal{P}(-1)<0$. On the other hand, if $\phi_{\pi}>\phi_{2}(\omega)$ and $\phi_{\pi}>1$ then from (50) we deduce that that $\mathcal{P}(1)<0$ and $\mathcal{P}(-1)>0$. Hence, under both cases, $\mathcal{P}(e)=0$ has one root inside the unit circle and the other one outside of it. Since there are two non-predetermined variables, we conclude that there exist multiple equilibria. Note that the roots are real as either $\mathcal{P}(1)<0$ or $\mathcal{P}(-1)<0$.

Part b2) is proved using (52) to derive that when $1<\phi_{\pi}<\phi_{2}(\omega)$, then $\operatorname{Trace}(\Omega-I)<0$ and $\operatorname{Det}(\Omega-I)>0$. Using these and the fact that under multiple equilibria the roots are real, we can apply part c) of Lemma 2 to conclude that the CF representation of stationary "sunspots" is E-stable.

To prove b3) recall that if $\phi_{y} \geq \frac{2}{\alpha}$ then $\phi_{3}(\omega)<1$. For the case $1<\phi_{\pi}<\phi_{2}(\omega)$ we have that the second expression in (52) implies that $\operatorname{Det}(\Omega-I)<0$. By part b) of Lemma 2 we conclude that the MSV solution is E-unstable.

Finally by simple differentiation we obtain $\frac{\partial \omega^{u}}{\partial \phi_{y}}<0$ and $\frac{\partial \phi_{3}(\omega)}{\partial \phi_{y}}<0$.

An active forward-looking rule that responds to expected sticky-price output restricts the determinacy and E-stability areas of Figure 1, in both the $\omega$ and $\phi_{\pi}$ dimensions. First, for mild responses $\left(0<\phi_{y}<\frac{2}{\alpha}\right)$, the $\omega^{\prime}$ s upper threshold-now denoted by $\omega^{u}$-is smaller than the $\omega^{*}$ identified in Propositions 1-3, and strictly decreasing in $\phi_{y}$. Moreover, for any $\omega \in\left(0, \omega^{u}\right)$, the $\phi_{\pi}$ 's upper bound $\phi_{3}(\omega)$ is always below the threshold $\phi_{1}(\omega)$ identified in Proposition 1 and indeed decreasing in $\phi_{y}$. Second, for a response to expected output above $\frac{2}{\alpha}$, the subset of active rules inducing a determinate and E-stable equilibrium is completely empty for any $\omega \in(0,1)$. In this case, the economy displays pervasive aggregate instability. ${ }^{37}$

The destabilizing effects of responding to output persist in the more general case studied in Subsection C. With both sectors featuring nominal rigidities, we allow the rule to respond to a measure of aggregate output. That is, $\widehat{r}_{t}=\phi_{\pi} \mathbb{E}_{t} \widehat{\pi}_{t+1}^{o}+\phi_{y} \mathbb{E}_{t} \widehat{y}_{t+1}^{o}$, with $\phi_{\pi}>1, \phi_{y}>0, \widehat{y}_{t+1}^{o}=\omega^{y} \widehat{y}_{t+1}^{F}+\left(1-\omega^{y}\right) \widehat{y}_{t+1}^{S}$ and $\omega^{y} \in[0,1]$ being some arbitrary weight. Using the log-linear versions of the CPI (3), the relative

\footnotetext{
${ }^{37}$ Following a similar logic, the benefits from an explicit positive response to current sticky-price output should be evident. The hybrid rule $\widehat{r}_{t}=\phi_{\pi} \mathbb{E}_{t}\left[\omega \widehat{\pi}_{t+1}^{F}+(1-\omega) \widehat{\pi}_{t+1}^{S}\right]+\phi_{y} \widehat{y}_{t}^{S}$ can be written as $\widehat{r}_{t}=\phi_{\pi} \mathbb{E}_{t} \widehat{\pi}_{t+1}^{S}+\phi_{\pi} \omega \theta \mathbb{E}_{t} \widehat{y}_{t+1}^{S}+$ $\left(\phi_{y}-\phi_{\pi} \omega \theta\right) \widehat{y}_{t}^{S}$. Then the effective response to current output could be positive for $\phi_{y}>\phi_{\pi} \omega \theta$. And, as suggested by Bullard and Mitra (2002), this positive response could make determinacy and E-stability more likely to occur. Results are available from the authors upon request.
} 

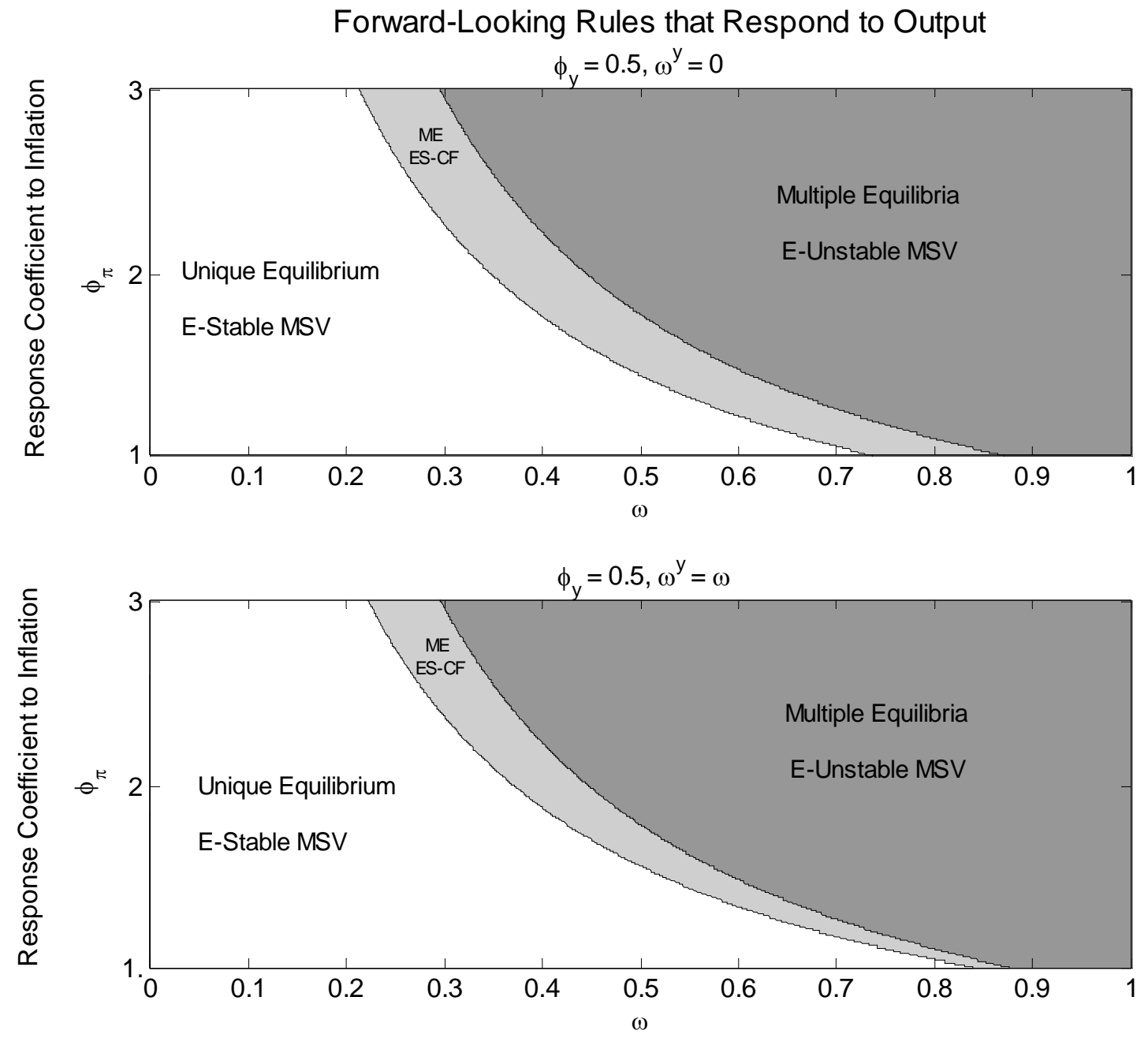

Weiaht on Flexible-Price Inflation

Figure 8: Regions of determinacy and expectational stability for forward-looking rules that also respond to output $\left(\phi_{y}>0\right)$. Goods are complemetns $\xi=0.5$. Parameters other than $\phi_{\pi}$ and $\omega$ are set at baseline values shown in Table 1.

Note: $\mathrm{ME}=$ Multiple Equilibria, ES-CF = E-Stable Common Factor representation of a "sunspot" equilibrium, and MSV = Minimum State Variable representation of a fundamental equilibrium. 
demands in (4) and the relative price $q_{t}=\frac{P_{t}^{F}}{P_{t}^{S}}$, after simple algebra, this extended rule can be written as: $^{38}$

$$
\begin{aligned}
\widehat{r}_{t}= & {\left[\phi_{\pi} \omega+\phi_{y} \xi\left(\eta-\omega^{y}\right)\right] \mathbb{E}_{t} \widehat{\pi}_{t+1}^{F}+\left[\phi_{\pi}(1-\omega)-\phi_{y} \xi\left(\eta-\omega^{y}\right)\right] \mathbb{E}_{t} \widehat{\pi}_{t+1}^{S} } \\
& +\phi_{y} \mathbb{E}_{t} \widehat{y}_{t+1}+\phi_{y} \xi\left(\eta-\omega^{y}\right) \widehat{q}_{t} .
\end{aligned}
$$

Figure 8 displays the results for the case of $\omega^{y}=0$ and $\omega^{y}=\omega$. That is, when the rule responds to stickier price output only, and the output weights are equal to the respective inflation weights. By comparing them with the bottom panel of Figure 2, we can notice that indeed a positive response to output aggravates aggregate instability problems, by enlarging both the area where the fundamental REE is not learnable and the area where there exist learnable "sunspot" equilibria.

The quantitative difference between the simple rule (19) and the extended rule (53) is further stressed in Table 3. By comparing the latter to Table 1, one can see that a mild response to output, $\phi_{y}=0.5$, lowers both the $\phi_{\pi}$ and the $\omega$ upper bounds, thus enlarging the indeterminacy/E-instability areas. For instance, a rule responding to the CPI inflation measure, $\omega=0.4$, with a coefficient $\phi_{\pi}=2$ induces a unique equilibrium under the simple rule, but learnable "sunspot" equilibria under the extended rule, for both $\omega^{y}=0$ and $\omega^{y}=\omega$.

Table 3: Augmented Forward-Looking Rules $\left(\phi_{y}=0.5\right)$ Determinacy and E-stability Bounds for Complement Goods

\begin{tabular}{l|c|c|c|c|c}
\hline \hline & \multicolumn{2}{|c|}{ Determinacy } & \multicolumn{3}{c}{ Learning } \\
\cline { 2 - 6 } & UE & ME & ES-MSV & ES-CF Sunspot & EU-MSV \\
\hline \hline & & & & & \\
a) $\omega^{c}=\omega$ & & & & \\
1) $\phi_{\pi}=2$ & $0 \leq \omega<0.38$ & $0.38<\omega \leq 1$ & $0 \leq \omega<0.45$ & $0.38<\omega<0.45$ & $0.45<\omega \leq 1$ \\
2) $\omega=\eta=0.4$ & $1<\phi_{\pi}<1.88$ & $1.88<\phi_{\pi}$ & $1<\phi_{\pi}<2.24$ & $1.88<\phi_{\pi}<2.24$ & $2.24<\phi_{\pi}$ \\
& & & & & \\
b) $\omega^{c}=0$ & & & & & \\
1) $\phi_{\pi}=2$ & $0 \leq \omega<0.35$ & $0.35<\omega \leq 1$ & $0 \leq \omega<0.45$ & $0.35<\omega<0.45$ & $0.45<\omega \leq 1$ \\
2) $\omega=\eta=0.4$ & $1<\phi_{\pi}<1.77$ & $1.77<\phi_{\pi}$ & $1<\phi_{\pi}<2.23$ & $1.77<\phi_{\pi}<2.23$ & $2.23<\phi_{\pi}$ \\
& & & & & \\
\hline
\end{tabular}

Note: $\omega^{*}=0.79$ for $\omega^{c}=\omega \cdot \omega^{*}=0.74$ for $\omega^{c}=0 . \mathrm{UE}=$ Unique equilibrim. ME $=$ Multiple Equilibria.

ES-MSV $=$ E-stable (learnable) MSV representation of a fundamental equilibrium.

ES-CF Sunspot = E-stable (learnable) CF representation of a "sunspot" equilibrium.

EU-MSV $=$ E-unstable (non-learnable) MSV representation of a fundamental equilibrium.

\footnotetext{
${ }^{38}$ Notice that for $\omega^{y}=\eta$ the rule (53) is equivalent to (19)-(20) with the term $\phi_{y} \mathbb{E}_{t} \widehat{y}_{t+1}$ added. On the other hand, for $\omega^{y}$ smaller (respectively, larger) than $\eta$, we would have a stronger (respectively, smaller) response to $\mathbb{E}_{t} \widehat{\pi}_{t+1}^{F}$ and a smaller (respectively, stronger) response to $\mathbb{E}_{t} \widehat{\pi}_{t+1}^{S}$.
} 


\section{D.2. The Cash-In-Advance Timing}

We explore now the consequences of introducing the cash-in-advance (CIA) timing in our model. To do this, we follow Carlstrom and Fuerst (2001) and assume that the money entering the utility function (1) is not $\frac{M_{t}}{P_{t}}$ but $\frac{N_{t}}{P_{t}}=\frac{M_{t-1}+R_{t-1} B_{t-1}-B_{t}+T_{t}^{g}}{P_{t}}$, where $P_{t}$ is the CPI defined in (3). We skip the derivation of the reduced form of the model and instead focus on the main difference between the two timings: the Euler equation for aggregate consumption.

Under the CIA timing, the log-linearized Euler Equation is

$$
\widehat{c}_{t}=\mathbb{E}_{t} \widehat{c}_{t+1}-\sigma^{-1}\left(\mathbb{E}_{t} \widehat{r}_{t+1}-\mathbb{E}_{t} \widehat{\pi}_{t+1}\right),
$$

where, in contrast to CWID timing that we adopted in the main text, the nominal interest rate is scrolled forward one period. For the general two-sector model studied in Subsection C, the equilibrium dynamics are described equations (32)-(34) together with this new Euler equation, and the interest rate rule.

It is straightforward to notice that the CIA Euler Equation with a contemporaneous rule is identical to the CWID Euler equation under a forward-looking rule. Hence, the determinacy and learnability conditions for a forward-looking rule under the CWID timing must be identical to those for a contemporaneous rule under the CIA timing. It is possible to prove this isomorphism for the dichotomous economy studied in Subsection B. In that case, the sticky-price consumption Euler equation (25) is replaced by $\widehat{c}_{t}^{S}-\mathbb{E}_{t} \widehat{c}_{t+1}^{S}=-\alpha\left(\mathbb{E}_{t} \widehat{r}_{t+1}-\mathbb{E}_{t} \widehat{\pi}_{t+1}^{S}\right)$, with the only difference being $\mathbb{E}_{t} \widehat{r}_{t+1}$ instead of $\widehat{r}_{t}$. The following proposition states this result.

Proposition 5 Consider the CIA model and the contemporaneous rule $\widehat{r}_{t}=\phi_{\pi} \widehat{\pi}_{t}^{o}$ with $\phi_{\pi}>1$ and $\omega \in(0,1)$. Then the determinacy and learnability conditions under which this rule induces a unique equilibrium, multiple equilibria, learnable or non-learnable fundamental equilibria, and learnable sunspots are the same as those stated in Propositions 1, 3, and 2 for a forward-looking rule in the CWID model.

Proof. It is sufficient to prove that, for the determinacy and learning analyses, the dynamic system of the CIA model for a contemporaneous rule is identical to the system of the CWID model for a forward-looking rule. To prove this, combine (6), (22), (28), the rule $\widehat{r}_{t}=\phi_{\pi} \widehat{\pi}_{t}^{o}$, and (54) to obtain the system (40) where $\widehat{x}_{t}=\left[\widehat{\pi}_{t}^{S}, \widehat{c}_{t}^{S}\right]^{\prime}, \Upsilon=[0,0]^{\prime}$ and $\Omega$ is defined as in (41). Then the conclusion follows. 


\section{References}

Airaudo, M., and L. F. Zanna, 2010, "On the Measure of Inflation for Interest Rate Rules with Credit Market Imperfections," Manuscript, Drexel University.

Adam K., and A. Marcet, 2010, "Internal Rationality, Imperfect Market Knowledge and Asset Prices," Manuscript, Mannheim University.

Anand, R., and E. Prasad, 2010, "Optimal Price Indices for Targeting Inflation Under Incomplete Markets," NBER Working Papers 16290.

Aoki, K., 2001, "Optimal Monetary Policy Responses to Relative-Price Changes," Journal of Monetary Economics, Vol. 48(1), pp. 55-80.

Azariadis, C., 1993, Intertemporal Macroeconomics. (Cambridge, Massachusetts: Blackwell).

Barsky, R. B., C. L. House, and M. S. Kimball, 2007, "Sticky-Price Models and Durable Goods," American Economic Review, Vol. 97(3), pp. 984-998.

Benhabib, J., S. Schmitt-Grohé, and M. Uribe, 2001, "Monetary Policy Rules and Multiple Equilibria," American Economic Review, Vol. 91(1), pp. 167-184.

Benigno, P., 2004, "Optimal Monetary Policy in a Currency Area," Journal of International Economics, Vol. 63(2), pp. 293-320.

Bils, M., and P. Klenow, 2004, "Some Evidence on the Importance of Sticky Prices," Journal of Political Economy, Vol. 112(5), pp. 947-985.

Blanchard, O., and C. Kahn, 1980, "The Solution of Linear Difference Models Under Rational Expectations," Econometrica, Vol. 48(5), pp. 1305-1312.

Bodenstein, M., C. J. Erceg, and L. Guerrieri, 2008, "Optimal Monetary Policy with Distinct Core and Headline Inflation Rates," Journal of Monetary Economics, Vol. 55, Supplement 1, pp. S18-S33.

Boivin, J., 2006, "Has U.S. Monetary Policy Changed? Evidence from Drifting Coefficients and Real-Time Data," Journal of Money, Credit and Banking, Vol. 38(5), pp. 1149-1174.

Bouakez, H., E. Cardia, and F. J. Ruge-Murcia, 2009, "The Transmission of Monetary Policy in a Multi-Sector Economy," International Economic Review, Vol. 50(4), pp. 1243-1266.

Bullard, J., and K. Mitra, 2002, "Learning About Monetary Policy Rules," Journal of Monetary Economics Vol. 49(6), pp. 1105-1129.

Canzoneri, M.R., R. Cumby, and B. Diba, 2003, "Recent Developments in the Macroeconomic Stabilization Literature: Is Price Stability a Good Stabilization Strategy?," in Dynamic Macroeconomic Analysis: Theory and Policy in General Equilibrium, ed. by S. Altug, J. Chadha, and C. Nolan. (Cambridge: Cambridge University Press).

Carlstrom, C. and T. Fuerst, 2001, "Timing and Real Indeterminacy in Monetary Models," Journal of Monetary Economics, Vol. 47(2), pp. 285-298.

Carlstrom, C., T. Fuerst, and F. Ghironi, 2006a, "Does It Matter (For Equilibrium Determinacy) What Price Index The Central Bank Targets," Journal of Economic Theory, Vol. 128(1), pp. 214-231.

Carlstrom, C., T. Fuerst, F. Ghironi, and K. Hernández, 2006b, "Relative Price Dynamics and the Aggregate Economy," Manuscript, Boston College.

Carvalho, C., 2006, "Heterogeneity in Price Stickiness and the Real Effects of Monetary Shocks," Frontiers of Macroeconomics, Vol. 2(1), Article 1. 
Carvalho, C. and N. Dam, 2010, "The Cross-sectional Distribution of Price Stickiness Implied by Aggregate Data," Manuscript.

Catao, L.A.V. and R. Chang, 2010, "World Food Price and Monetary Policy," IMF Working Paper 10/161.

Clarida, J., J. Galí, and M. Gertler, 2000, "Monetary Policy Rules and Macroeconomic Stability: Evidence and Some Theory," Quarterly Journal of Economics, Vol. 115(1), pp. 147-180.

Cochrane, J.H., 2009, "Can Learnability Save New-Keynesian Models?," Journal of Monetary Economics, Vol. 56(8), pp. 1109-1113.

Cogley, T., and T. J. Sargent, 2005, "Drifts and Volatilities: Monetary Policy and Outcomes in the Post WWII US," Review of Economic Dynamics, Vol. 8(2), pp. 262-302.

Dhawan, R., and K. Jeske, 2007, "Taylor Rules and Headline Inflation: A Bad Idea," Working Paper 2007-14, Federak Reserve Bank of Atlanta.

Eusepi, S., 2007, "Learnability and Monetary Policy: a Global Perspective," Journal of Monetary Economics, Vol. 54(4), pp. 1115-1131.

Evans, G. W. and S. Honkapohja, 2001, Learning and Expectations in Macroeconomics, (Princeton: Princeton University Press).

Evans, G. W., and S. Honkapohja, 2003, "Adaptive Learning and Monetary Policy Design," Journal of Money, Credit and Banking, Vol. 35(6), pp. 1045-1072.

Evans, G. W. and B. McGough, 2005, "Monetary Policy, Indeterminacy and Learning," Journal of Economic Dynamics and Control, Vol. 29(11), pp. 1809-1840.

Galí, J. and T. Monacelli, 2005, "Monetary Policy and Exchange Rate Volatility in a Small Open Economy," Review of Economic Studies, Vol. 72(7), pp. 707-734.

Honkapohja, S., K. Mitra, and G. W. Evans, 2003, "Notes on Agents' Behavioral Rules Under Adaptive Learning and Recent Studies of Monetary Policy," Manuscript, University of St. Andrews.

Klenow, P.J., and O. Kryvtsov, 2008, "State-Dependent or Time-Dependent Pricing: Does It Matter for Recent U.S. Inflation?," Quarterly Journal of Economics, Vol. 123(3), pp. 863-904.

Klenow, P.J., and B. A. Malin, 2010, "Microeconomic Evidence on Price Setting," NBER, Working Papers 15826.

Llosa, G., Tuesta, V., 2008. Determinacy and Learnability of Monetary Policy Rules in Small Open Economies. Journal of Money, Credit and Banking, Vol. 40(5), 1033-1063.

Llosa, G., and V. Tuesta, 2009, "Learning about Monetary Policy Rules when the Cost Channel Matters," Journal of Economic Dynamics and Control, Vol. 33(11), pp. 1880-1896.

Mankiw, G. and R. Reis, 2003, "What Measure of Inflation Should a Central Bank Target?," Journal of European Economic Association, Vol. 5(9), pp. 1058-1086.

Marcet, A and T. Sargent, 1989, "Convergence of Least-Square Learning Mechanisms in Self-Referential Linear Stochastic Models," Journal of Economic Theory, Vol. 48(2), pp. 337-368.

Mishkin, F.S., 2008, "Does Stabilizing Inflation Contribute to Stabilizing Economic Activity?," NBER Working Paper 13970.

McCallum, B., 1983, "On Nonuniqueness in Linear Rational Expectations Models: An Attempt at Perspective," Journal of Monetary Economics, Vol. 11(2), pp. 134-168.

McCallum, B.T., 2009, "Inflation Determination with Taylor Rules: Is New-Keynesian Analysis Critically Flawed?," Journal of Monetary Economics, Vol. 56(8), pp. 1101-1108. 
Nakamura, E. and J. Steinsson, 2008, "Five Facts About Prices: A Reevaluation of Menu Costs Models," Quarterly Journal of Economics, Vol. 123(4), pp. 1415-1464.

Preston, B., 2006, "Adaptive Learning, Forecast-based Instrument Rules and Monetary Policy," Journal of Monetary Economics, Vol. 53(3), pp. 507-535.

Ravenna, F. and C. Walsh, 2006, "Optimal Monetary Policy with the Cost Channel," Journal of Monetary Economics, Vol. 53(2), pp. 199-216.

Rotemberg, J., 1982, "Sticky Prices in the United States," Journal of Political Economy, Vol. 90(6), pp. 11871211.

Schorfheide, F., 2005, "Learning and Monetary Policy Shifts," Review of Economic Dynamics, Vol. 8(2), pp. $392-419$.

Svensson, L. and N. Williams, 2007, "Bayesian and Adaptive Optimal Policy under Model Uncertainty," NBER Working Papers 13414.

Taylor, J. B., 1993, "Discretion Versus Policy Rules in Practice," Carnegie-Rochester Conference Series on Public Policy, Vol. 39(1), pp. 195-214.

Taylor, J., 1999, "A Historical Analysis of Monetary Policy Rules," in Monetary Policy Rules, ed. by J. Taylor. (Chicago: University of Chicago Press).

Woodford, M., 2003. Interest and Prices: Foundations of a Theory of Monetary Policy. (Princeton: Princeton University Press).

Wynne, M.A., 2008, "How Should Central Banks Define Price Stability?," Working Paper No.8, Globalization and Monetary Policy Institute, Federal Reserve Bank of Dallas. 